\title{
Age-associated changes to neuronal dynamics involve a loss of inhibitory signaling in $C$. elegans
}

Short Title: Age-related loss of inhibitory signaling in C. elegans.

Authors: Gregory S Wirak BS

Jeremy Florman PHD

Mark J Alkema PHD

Christopher W Connor MD PHD

Christopher V Gabel PHD

Affiliation:

(Wirak)

PhD Candidate

Graduate Program for Neuroscience

Department of Physiology and Biophysics

Boston University School of Medicine, Boston, MA, USA

(Florman) Postdoctoral Researcher

Department of Neurobiology

University of Massachusetts Medical School, Worcester, MA, USA

(Alkema) Professor of Neurobiology

University of Massachusetts Medical School, Worcester, MA, USA

(Connor) Assistant Professor of Anesthesiology

Department of Anesthesiology, Perioperative and Pain Medicine

Brigham and Women's Hospital, Boston, MA, USA

Research Associate Professor of Physiology and Biophysics

Boston University School of Medicine, Boston, MA, USA

(Gabel) Associate Professor of Physiology and Biophysics

Boston University School of Medicine, Boston, MA, USA

\section{Corresponding Author:}

Dr. Christopher V Gabel

Department of Physiology and Biophysics

Boston University School of Medicine

700 Albany Street, W302C

Boston, MA 02118

Email: $\quad$ cvgabel@bu.edu 
bioRxiv preprint doi: https://doi.org/10.1101/2021.07.07.451497; this version posted July 8, 2021. The copyright holder for this preprint (which was not certified by peer review) is the author/funder. All rights reserved. No reuse allowed without permission.

\section{Financial Support:}

NIH R01 GM121457

NIH R01 NS107475

NIH T32 GM008541

Departmental support

Paper Type: Original Investigation 


\section{ABSTRACT}

In the aging brain, many of the alterations underlying cognitive and behavioral decline remain opaque. $C$. elegans offers a powerful model for aging research, with a simple, well-studied nervous system to further our understanding of the cellular modifications and functional alterations accompanying senescence. We perform multi-neuronal functional imaging across the aged $C$. elegans nervous system, measuring an age-associated breakdown in system-wide functional organization. At single-cell resolution, we detect shifts in activity dynamics toward higher frequencies, alongside a specific loss of inhibitory signaling occurring early in the aging process. These effects are partially delayed or accelerated by a long-lived or neurodegenerative mutant background, respectively. We further provide evidence that these effects are partially mediated through degradation of GABA signaling, via a pathway involving UNC-2/CaV2 $\alpha$ and caspase activation. Data from mammals are consistent with our findings, suggesting a conserved shift in the balance of excitatory/inhibitory signaling with age leading to functional decline.

\section{INTRODUCTION}

Progressive breakdown of neuronal function is a hallmark of natural aging. Yet the relationship between the molecular, cellular, and ultrastructural modifications (Mattson and Arumugam, 2018), the alterations in neuronal dynamics and signaling, and the deficits in cognitive performance and behavior that emerge with advanced age remain unclear. At the cellular level, dramatic neuronal loss is not observed; however, changes in neuronal excitability and signaling have been well documented (Peters et al., 2008) (Morrison and Baxter, 2012). These effects include decreased inhibition within multiple sensory systems (Richardson et al., 2013) (Schmidt et al., 2010) (David-Jürgens and Dinse, 2010) (Cheng and Lin, 2013) and the hippocampus (Potier et al., 2006), suggesting a potential shift in the balance of excitatory/inhibitory signaling with age. Maintenance of this signaling balance is critical for functional homeostasis in the nervous system and its breakdown is associated with numerous pathological states, including autism spectrum disorders, epilepsy, and Alzheimer's disease (Turrigiano and Nelson, 2004) (Isaacson and Scanziani, 2011) (Vico Varela et al., 2019). While such imbalances might also contribute to shifts in functional organization with age, the link between alterations in cellular signaling and breakdown of system dynamics is ill-defined. Recent studies employing task-free functional magnetic resonance imaging ( $\mathrm{fMRI}$ ) have shown that aging alters intrinsic brain connectivity and the functional organization of large-scale resting state networks (Sala-Llonch et al., 2015), which correlates with diminished cognitive performance (Bagarinao et al., 2019) (Varangis et al., 2019). To further elucidate the link between age-associated decline in system-state dynamics and changes in activity and signaling at the cellular level, we turned to comprehensive system-wide functional neuronal imaging within the nematode C. elegans.

Recent advances in multi-neuron fluorescence imaging present an unprecedented ability to measure and understand the dynamics, function, and breakdown of small neuronal systems. Applied to the microscopic nematode Caenorhabditis elegans, such techniques enable measurement of neuronal activity across the majority of the nervous system with single cell resolution, revealing spontaneous behavioral state-dynamics (Kato et al., 2015) (Nguyen et al., 2016) (Venkatachalam et al., 2016) that are lost under unique circumstances, such as developmental quiescence or exposure to volatile anesthetics (Nichols et al., 2017) (Awal et al., 2020). Moreover, C. elegans is also a powerful system for the study of aging with longevity studies contributing much to our understanding of conserved molecular pathways affecting organismal aging (Mack et al., 2018). C. elegans is both short-lived and genetically tractable, facilitating the high-throughput analyses of how these pathways and their manipulation affect various aspects of normal aging. With its capabilities for comprehensive multi-neuron imaging and well-established aging studies, C. elegans presents a unique window into the age-associated breakdown of neuronal signaling and system dynamics.

Neuronal aging studies in C. elegans illustrate that its nervous system, comprised of 302 stereotyped and identifiable neurons, is largely spared from large anatomical age-related deterioration, paralleling what is seen in higher organisms. Gross morphology is well-preserved, with limited neurite deterioration (Herndon et al., 
2002), no neural apoptosis or necrosis (Pan et al., 2011), and no significant changes to the nuclear architecture (Haithcock et al., 2005). Closer inspection of neurite ultrastructure, however, revealed novel agedependent features, including ectopic outgrowths and the beading and blebbing of axons (Pan et al., 2011) (Tank et al., 2011) (Toth et al., 2012). A breakdown in synaptic integrity was also found to accompany aging, with evidence suggesting that functional neuronal decline precedes that of the musculature. Initial shifts in behavior, several of which correlate with reduced synaptic integrity, may therefore reflect nervous system deterioration rather than sarcopenia, although disentangling the two has been challenging (Toth et al., 2012) (Liu et al., 2013) (Mulcahy et al., 2013). The disruption of specific behaviors in the context of normal worm aging has also been well documented and includes behaviors ranging from defecation (Croll et al., 1977) (Bolanowski et al., 1981) (Felkai et al., 1999), crawling dynamics (Glenn et al., 2004) (Podshivalova et al., 2017), chemotaxis (Glenn et al., 2004), to associative learning (Kauffman et al., 2010). To date, however, studies that have directly examined neuronal activity underlying such behavioral changes have been limited to individual neurons and synapses (Chokshi et al., 2010) (Mulcahy et al., 2013) (Liu et al., 2013) (Zullo et al., 2019) (Huang et al., 2020) or simple circuits (Leinwand et al., 2015).

Here we perform functional multi-neuron fluorescence imaging in C. elegans throughout its lifespan. As the animals age, we measure a distinct loss of inhibitory signaling that alters the excitatory/inhibitory balance of the nervous system and is accompanied by an increase in individual neuron activity. These cellular effects correspond with a breakdown of system-wide behavior state dynamics. We determine that these effects may be partially mediated through a UNC-2/CaV2 calcium/caspase pathway known to degrade inhibitory GABA signaling during development. Finally, we show that a long-lived or neurodegenerative mutant background delays or accelerates many aspects of normal neuronal aging respectively. Our findings in C. elegans recapitulate numerous aspects of neuronal aging found in higher organisms, emphasizing the loss of inhibitory signaling and disruption of excitatory/inhibitory balance as a key element of neuronal decline and begin to uncover the cellular mechanisms driving these changes.

\section{RESULTS}

\section{Command interneuron AVA becomes more active and exhibits slower dynamics with age}

As the nematode C. elegans ages, the locomotive behavior of the animal is grossly altered (Fig1A). Young adult worms tend to crawl in a forward direction with infrequent bouts of spontaneous backward movement (reversals), which typically precede changes in the direction of travel and constitute an important component of the animal's foraging strategy (Pierce-Shimomura et al., 1999) (Croll, 1975) (CROLL, 1975). As has been previously shown (Glenn et al., 2004) (Podshivalova et al., 2017), we measure a progressive increase in the rate at which animals initiate reversals with age (Fig1B). We further observe that the typical reversal becomes shorter, resulting in the aged animal making more brief or "hesitant" reversals. While a reversal can be elicited by anterior mechanosensation (Chalfie et al., 1985), the age-dependent increase in reversal frequency cannot be explained by an enhancement in anterior touch sensitivity. Instead we measure a decrease in touch sensitivity with age (Fig1C), consistent with previous reports (Vayndorf et al., 2016). Therefore, this behavioral shift likely arises due to age-related changes in neuronal dynamics within the neurocircuitry controlling reversal behavior.

To determine the effects of aging on the command interneurons controlling $C$. elegans crawling, we performed functional fluorescence imaging of the premotor interneuron AVA, known to mediate backward locomotion (Chalfie et al., 1985) (Kawano et al., 2011) (Fig1D). AVA calcium transient traces measured as GCaMP fluorescence (Fig1E) were acquired from worms spanning the animals adult life i.e. days 1, 3, 6, 9, and 12 of adulthood. In healthy young animals, the AVA interneuron displays binary dynamics as it switches between active and inactive states. These states have been shown to correlate closely with reverse and forward crawling behavior, respectively (Chalfie et al., 1985) (Kawano et al., 2011). In aged worms, a striking reduction was observed in the rate at which AVA transitions from inactive to active state (i.e. from low to high GCaMP6s fluorescence). This is made visually apparent by overlaying and averaging the individual calcium transient 
onsets (Fig1F). Transient rise times were calculated as the time required to shift from $5 \%$ to $95 \%$ of the maximum fluorescence (as previously described (Wirak et al., 2020), see Statistical Methods). A progressive increase in AVA Rise Time is observed through days 1-12 of nematode adulthood (Fig1G). In contrast, the Fall Time (the time required to shift from $95 \%$ to $5 \%$ of the maximum fluorescence) shows no significant change with age (Fig1F\&H). Notably, the proportion of time AVA exhibits high activity (i.e. the duty ratio) progressively increases with age (Fig1I). This is primarily due to an increase in calcium transient duration, while the frequency of calcium transients remains unchanged (FigS1). Indeed, prolonged AVA activation is congruent with the increased tendency for reversals in aged animals.

\section{Global neuronal activity becomes increasingly disorganized with age}

We next sought to assess system-wide neuronal dynamics across the $C$. elegans lifespan. Using methods we recently developed, we employed light-sheet microscopy to simultaneously capture the GCaMP6s signal from 120 neurons within the $C$. elegans head-region (Awal et al., 2020). Measurements were acquired from worms at days $1,3,6,9$, and 12 of adulthood with 10-20 animals per condition. We observed substantially more noise at day 12, so experiments focused on day 9 as a robust senescent timepoint. Example results from individual animals at day 1 and day 9 are shown in Figure 2A, while additional day 1, 3, 6, 9 and 12 examples are displayed in Supplementary Figure S2. As we and others have observed previously (Kato et al., 2015), young adult animals display a clear system-wide organization of neuronal dynamics, with large groups of neurons showing correlated activity (Fig2A). The activity of such multi-neuron systems can be effectively presented using Principal Component Analysis (PCA), where the first three principal components (typically representing $80-90 \%$ of the variation in the system) are visualized in a 3-dimensional plot. As illustrated in Figure $2 \mathrm{C}$ and S2, neuronal activity in young adult animals typically traces out a smooth trajectory in such PCA plots, which constitutes a well-defined activity manifold. Prior studies have demonstrated that regions along these traces represent particular behavioral states of the animal (e.g. forward or backward movement or turning) that are linked by smooth transitions between states (Kato et al., 2015) (Awal et al., 2020).

As $C$. elegans age, we observe a striking breakdown in the system organization. The lower panel of Figure $2 \mathrm{~A}$ displays typical neuron activity measured in day 9 old animals. Apparent transitions between global states remain discernable by eye within the 120-neuron activity arrays. However, these states are less distinct and appear prolonged. This change is reflected in the accompanying PCA plot in the lower panel of Figure $2 \mathrm{C}$. In contrast to the smooth transitions between repeating neuronal states observed in the young animal, this plot displays a much more erratic trajectory, with no apparent overall organization. The contrast between neuronal dynamics of young and old worms is further exemplified by the time correlation plots (Fig2B, S2). The neuronal activity at each time point is compared to that at every other time point by calculating the relative distance in activity space (see Methods). Blue represents two time points with very similar neuronal activity patterns while red indicates significantly different neuronal activity patterns. In the young animal, there are a series of rapid transitions away from the reference activity pattern (yellow, red regions) and also transitions back to similar activity patterns (green, blue regions). In the older animal these dynamics break down. Transitions between states are slower and more erratic. Additionally, the system does not readily return to previous activity patterns, indicating a breakdown in the recurrence of these system states.

To further quantify the breakdown in system-wide dynamics, we measured the smoothness of the PCA trajectories over time. At each time point we calculated the absolute change in direction as the discrete time derivative of the tangential angle describing the trajectory. A smoothly curving trajectory will have small angular changes in direction over time, while a completely stochastic, randomized trajectory will have equally distributed changes in direction over the possible 180 degrees. Example probability histograms of angular changes for individual worms are displayed in Figure 1C inset and Figure S2. Pooling all such measurements from all trials taken at a particular age we generate an aggregate probability histogram of the angular directional changes of PCA trajectory for each age (Fig2D). For young adult day 1 animals, this histogram is highly skewed toward smaller angles, reflecting the smooth characteristic of the trajectories and temporal continuity in the neuronal activity. As the animals age this bias is progressively lost, generating histograms with more equal distributions across all angular changes reflecting erratic randomly changing trajectories. This 
increased stochasticity of the PCA traces across the population of animals with age reflects the gradual loss of organization and temporal continuity in neuronal activity.

\section{Aged neurons exhibit higher frequency activity and a specific loss of anti-correlativity}

Within each ensemble of recorded neurons, it is possible to measure how individual neuron dynamics change with age. Power spectral density (PSD) analyses have been extensively used to quantitatively describe neuronal activity (Draguhn and Buzsáki, 2004) (Buzsáki and Watson, 2012). We generated normalized PSDs for each of the 120 neurons imaged in each animal and averaged across all neurons measured within each age group (see Statistical Methods). Overlaying these average spectra revealed a progressive shift in relative power from low- to higher-frequencies with age (prominently from $0-0.05$ to $0.10-0.15 \mathrm{~Hz}$, respectively) (Fig3A) This shift is further illustrated by plotting each age group's cumulative power spectrum (Fig3A, insert). To quantify this effect, we calculated the $40 \%$ spectral edge for each worm's PSD (i.e. the frequency below which $40 \%$ of the power resides, see Statistical Methods). We observed a progressive increase in the average PSD $40 \%$ spectral edge with age (Fig3B).

The $C$. elegans nervous system possesses a subset of strongly connected neurons displaying highly correlated or anti-correlated activities (Cook et al., 2019). Such connections are essential for the effective organization and function of a nervous system. To measure changes in neuronal connectivity with age, we calculated the signal correlativity between all possible neuron pairs among the 40 most dynamically active neurons in each animal. We then pooled all correlation measurements at each age to generate an aggregate probability histogram of neuron-neuron correlations (Fig3C). We observe a striking reduction in the proportion of strongly anti-correlated neuron pairs with age. In contrast, the proportion of highly correlated neuron pairs remains relatively stable. To quantify this effect, we calculated the proportion of neuron pairs demonstrating strong negative or positive correlativity (i.e. the Negative and Positive Correlation Proportion, respectively) for each worm and averaged by age (see Statistical Methods). We observe a significant reduction in the Negative Correlation Proportion occurring as early as day 3 of adulthood and further progressing with age (Fig3D). However, no change is observed in the Positive Correlation Proportion (Fig.3E). The loss of neuronal pairs that are negatively correlated suggests a specific breakdown in inhibitory signaling with age, accompanied by a shift in the excitatory/inhibitory balance of the nervous system.

\section{Age-associated changes to neuronal dynamics are recapitulated in young unc-2 gain-of-function mutant animals.}

To investigate the molecular mechanisms that could underlie age-related changes in network behavior, we considered candidate genes known to regulate excitatory/inhibitory balance. Mutations in the unc-2/CaV2 $\alpha$ gene have been shown to alter the excitatory/inhibitory signaling balance in the $C$. elegans nervous system (Huang et al., 2019). unc-2 encodes the C. elegans orthologue of the pore-forming alpha-1A subunit of the voltage-dependent P/Q-type calcium channel (CACNA1A). Animals with the gain-of-function (gf) mutation unc2(zf35) show an increased rate of spontaneous reversals as young adults (Fig4A), strikingly similar to that of aged wild-type animals. Multi-neuron imaging in gain-of-function unc-2(gf) animals, revealed striking similarities between the neuronal dynamics of day 1 unc-2(gf) animals and those of older (day 9) wild-type animals. The average power spectra of this mutant displays the shift from low- to high-frequency power that accompanies wild-type aging as early as day 1 (Fig4B). Indeed, the $40 \%$ spectral edge is significantly increased at day 1 in unc-2(gf) animals, relative to wild-type (Fig4C). The distribution of neuron-pair correlations was similarly affected by the mutation, as the age-related changes were recapitulated at a younger age in unc-2(gf) animals (Fig4D). The Negative Correlation Proportion is significantly decreased at day 1 in unc-2(gf) animals, relative to wild-type (Fig4E), while the Positive Correlation Proportion is unchanged across ages and genotypes (data not shown). Likewise, the temporal continuity of system activity in unc-2(gf) animals breaks down at an early age, with the probability histogram of angular directional changes of PCA trajectory from day 1 unc-2(gf) worms closely matching that of day 9 wild-type animals (Fig4F). These experiments demonstrate that UNC-2/CaV2 $\alpha$ gain-of-function results in increased crawling reversal rate and neuronal hyperactivity, a loss of inhibitory 
signaling, and disruption of system organization in young animals, all of which closely match the effects observed with aging.

We next examined the canonical loss-of-function unc-2(e55) mutant strain recently shown to decrease the neuronal excitatory/inhibitory balance in C. elegans (Huang et al.). Young adult unc-2(If) worms show lethargic crawling behavior and a decreased spontaneous reversal rate throughout their lifespan (Fig4A). We performed multi-neuronal imaging on the unc-2(If) animals at day 1 and day 9 of adulthood. We find that the PSD of unc2(If) animals shifts to higher frequencies to a far lesser extent with age (Fig4B\&C). Likewise, the $40 \%$ spectral edge is trending lower at day 9 , relative to wild-type $(p=0.06)$ (Fig4C). However, age-related changes to the neuron-pair correlativity appear unaffected (Fig4D), as the Negative Correlation Proportion at day 9 remains comparable to wild-type (Fig4E). Moreover, the breakdown in temporal continuity of system activity appears largely unchanged with only a slight shift in the distribution of PCA angular directional changes at day 9 compared to wild-type (Fig4F). Thus, UNC-2/CaV2 $\alpha$ loss-of-function appears to preserve many, but not all aspects, of neuronal signaling that are typically altered with age.

\section{Age-associated changes to neuronal dynamics are partially rescued in aged ced-4 loss-of-function mutants}

During development, UNC-2 mediated calcium signaling has been directly linked to the removal of presynaptic GABAergic complexes in C. elegans motor neurons (Miller-Fleming et al., 2016). This signaling triggers CED-3 caspase activity to degrade these presynaptic domains and is blocked by a loss-of-function mutation in its upstream regulator CED-4/Apaf-1 (Miller-Fleming et al., 2016). To test if a similar process may be at work during aging, we tested a loss-of-function ced-4(n1162) mutant strain in our imaging assays. In these animals we did not observe the characteristic shift from low to high frequency in the neuronal activity PSD, resulting in no significant change in the $40 \%$ spectral edge with age (Fig5A\&B). Likewise, the loss of strongly anticorrelated neuron pairs with age was diminished (Fig5C). The Negative Correlation Proportion in ced-4 worms is not significantly changed with age, when comparing days 1 and 9 , and is significantly higher on day 9 , as compared to wild-type day 9 (Fig5D). Similar to the unc-2(If) mutant, the age-dependent breakdown in systemwide temporal continuity was largely unchanged in ced-4 animals, with only a very mild shift in the distribution of PCA angular directional changes at day 9 compared to wild-type (Fig5E). These results show striking similarities to that of loss-of-function unc-2(If), suggesting that they may indeed contribute to the same cellular processes affecting neuronal aging.

\section{Agonizing $\mathrm{GABA}_{\mathrm{A}}$ receptors partially restores neuronal dynamics in aged worms}

To verify that a breakdown in inhibitory GABA signaling plays an important role in the age-dependent changes to neural dynamics, we applied the $\mathrm{GABA}_{\mathrm{A}}$ agonist muscimol to young adult and aged worms. We found that acute muscimol application reshapes the neuronal activity PSD in a manner opposite to the effects of aging, causing a shift from high to low frequency power in both young adult and aged animals and significantly lowering the $40 \%$ spectral edge in day 9 animals (Fig6A\&B). Unsurprisingly, there is no effect on the agedependent loss of negative correlation between neurons in muscimol-treated animals (Fig6C\&D), as increased inhibitory tone from an exogenous ligand should not affect neuronal connectivity. However, muscimol treatment did somewhat rescue system organization in the aged animal, as PCA analysis reveals better temporal continuity in neuronal dynamics (i.e. the distribution of PCA angular directional changes retains more bias toward smaller angles in day 9 animals) (Fig6E). Taken as a whole, these results demonstrate that pharmacological stimulation of $\mathrm{GABA}_{A}$ signaling can counteract many of the age-dependent effects we observe in C. elegans neuronal dynamics.

\section{Long-lived daf-2 and neurodegenerative sel-12 mutations alter neuronal dynamics with age.}

To further assess the effects of aging on neuronal dynamics, we examined a genetic strain with extended lifespan, as well as a strain displaying age-associated neurodegeneration. C. elegans harboring a loss-of-function mutation within the insulin/ IGF-1 receptor orthologue daf-2 display robust longevity (Kenyon et al., 1993). daf- 
2 reversal behavior is curious, as young adults have been shown to display a high reversal rate that is maintained with age (FigS3A) (Podshivalova et al., 2017). Imaging the AVA neuron individually, we find that AVA activation and inactivation dynamics are fully preserved at day 9 (FigS3B-D), but the interneuron's duty ratio is still altered with age, similar to wild-type. (FigS3E). Performing multi-neuronal imaging, we find that daf2 mutation appears to prevent or delay some but not all characteristics of normal aging. The shift in neuronal activity towards higher frequencies (Fig7A) appears to be diminished, with the $40 \%$ spectral edge of day 9 daf2 worms showing no significant difference from day 1 daf-2 and trending lower than day 9 wild-type animals ( $p$ $=0.13)$ (Fig7B). Likewise, age-dependent breakdown in system-wide temporal continuity was somewhat diminished in daf-2 animals, with the distribution of PCA angular directional changes retaining more bias toward smaller angles (Fig7l). However, the loss of strongly anti-correlated neuron pairs with age was unaffected by daf-2 mutation, with the Negative Correlation Proportion changing with age comparably to wildtype (Fig7E\&F).

Mutations in the C. elegans orthologue to presenilin PSEN2, sel-12, were recently shown to display functional and anatomical neurodegeneration during early life (Sarasija et al., 2018). Performing multi-neuronal imaging, we found the average neuronal activity PSD of young day 1 sel-12 worms is highly similar to that of aged day 9 wild-type animals (Fig7C). Young sel-12 animals also display decreased anti-correlativity between neuronpairs, and a reduced Negative Correlation Proportion, similar to that of day 9 wild-type animals (Fig7G\&H). Interestingly, they also display a reduction in strongly correlated neuron pairs that we do not see in wild-type at any age, (Fig7G). Finally, we found a large effect on system-wide dynamics and temporal continuity, with the distribution of PCA angular directional changes for sel-12 day 1 and day 3 animals closely matching that of day 9 wild-type animals (Fig7J). These findings demonstrate distinct parallels in the neuronal dynamics in the context of sel-12 neurodegeneration and that of healthy neuronal aging.

\section{DISCUSSION}

Despite the simplicity of the $C$. elegans nervous system, it has demonstrated the capacity to mediate a variety of complex behaviors, such as chemotaxis and associative learning, which exhibit age-associated decline (Glenn et al., 2004) (Kauffman et al., 2010). We are beginning to gain insight into the alterations of the nervous system that underlie this functional decline with age, including changes to neurite ultrastructure (Pan et al., 2011) (Tank et al., 2011) (Toth et al., 2012), synaptic transmission (Liu et al., 2013) (Mulcahy et al., 2013), and basal activity and excitability of specific neurons (Chokshi et al., 2010) (Liu et al., 2013) (Leinwand et al., 2015) (Zullo et al., 2019) (Huang et al., 2020). In this study, we expand these efforts to include advanced multineuron fluorescence imaging that captures the activity dynamics across large swaths of the nervous system at single-cell resolution. Measuring global neural activity in aged $C$. elegans revealed novel age-associated changes in neuronal signaling and activity that underlie the decline in system dynamics and organization.

With age, we observe a striking breakdown in system-wide organization and temporal continuity of neuronal dynamics. This decline includes a loss of well-defined and recurrent system states observed in young adult animals that can be visualized using both principal component trajectories (Fig2C, S2) and time correlation heatmaps (Fig2B, S2). While young worms display bouts of rapid transitions between repeated states, old worms appear to dwell for longer periods of time in less-defined states, transition slowly between states, and do not efficiently return to prior states. These age-associated changes in global system dynamics are congruent with the prolonged calcium transients and slower state transitions we observe in the AVA command interneuron (Fig1F-I). Moreover, the principal component trajectories of older animals display progressively more random, erratic changes in direction over short time-periods, as measured by the distribution of angular changes (Fig2D). These observations are reflective of a system that has lost temporal continuity and no longer adheres to a well-defined activity manifold. Interestingly, the most dramatic changes in system organization occur as early as day 3 of adulthood, suggesting that breakdown of system-wide state dynamics begins remarkably early in the aging process. 
We observe a progressive reduction in anti-correlated neuronal activity with advancing age in C. elegans (Fig3C). However, this is not accompanied by a loss of positive correlativity, resulting in a specific loss of inhibitory signaling with age that shifts the of excitatory/inhibitory balance toward excitation (Fig3D\&E). Again, these effects begin as early as day 3 of adulthood. Maintaining an appropriate excitatory/inhibitory balance is essential to the health and function of the nervous system. Increased excitability has been associated with aging in higher order species (Richardson et al., 2013) (Schmidt et al., 2010) (David-Jürgens and Dinse, 2010) (Cheng and Lin, 2013) (Potier et al., 2006), and also many neurological diseases, such as Alzheimer's disease (Vico Varela et al., 2019), migraines (Vecchia and Pietrobon, 2012), and epilepsy (Fritschy, 2008). We also observe a concurrent increase in neuronal activity in aged worms, measured as a shift toward higher frequency dynamics (Fig3A\&B). Interestingly, this shift in frequency power occurs primarily after the loss of inhibitory signaling, sometime between day 3 and 6 of adulthood. The clinical relevance of such findings is highlighted by a recent study that ties decreased neuron excitability to longevity, both in humans and C. elegans (Zullo et al., 2019). Our results here demonstrate that shifts in the excitatory/inhibitory balance in C. elegans with age are not due to increased excitatory signaling but rather a distinct loss of inhibitory signaling.

In C. elegans, the presynaptic voltage-gated calcium channel UNC-2/CaV2 $\alpha$ has been shown to modulate the excitatory/inhibitory balance of the nervous system. Specifically, unc-2 gain-of-function (gf) has a differential effect on excitatory versus inhibitory signaling at the neuromuscular junction, with increased cholinergic signaling and reduced GABAergic signaling (Huang et al., 2019). Moreover, young unc-2 gain-of-function mutants display multiple characteristics similar to those of aged wild-type worms. These mutants initiate spontaneous reversals more frequently than their wild-type counterparts (Fig4A) (Huang et al., 2019) and also demonstrate an increased sensitivity to aldicarb (a nematode cholinesterase inhibitor), reflecting an increase in neuromuscular acetylcholine signaling (Huang et al., 2019). These characteristics are also observed in aged $C$. elegans (Glenn et al., 2004) (Podshivalova et al., 2017) (Mulcahy et al., 2013). Upon examining young day 1 unc-2 gain-of-function worms using our own imaging techniques, we observe a breakdown in system organization and shifts in neural dynamics that are strikingly similar to those in wild-type day 9 senescent animals. This is true across all metrics: spectral power (Fig4B\&C), neuronal correlativity (Fig4D\&E), and system organization as measured by the angular changes in principal component trajectory (Fig4F). Meanwhile, in unc-2 loss-of-function (If) mutants, we observe a partial rescue of such age-associated changes, primarily with respect to spectral power (Fig4B\&C).

Huang et al. demonstrated that unc-2 gain-of-function leads to a reduction in GABAergic signaling at the neuromuscular junction. Moreover, they found that these mutant animals were partially resistant to the GABA agonist muscimol (Huang et al., 2019). Mammalian studies have shown diminished prominence and functional integrity of inhibitory $\gamma$-aminobutyric acid (GABA) synapses with age (McQuail et al., 2015) (Rozycka and Liguz-Lecznar, 2017). We therefore assessed the effects of acute muscimol exposure in senescent wild-type worms. We find that application of the drug reshapes the power spectral density in a manner antithetical to the effects of aging, restoring low frequency power and reducing high frequency power in aged day 9 worms. Interestingly, muscimol has a comparable effect on young day 1 animals (Fig6A\&B). Muscimol application also partially restores system-wide organization and temporal continuity in day 9 worms (Fig6E). While we do not observe any effects on neuronal correlativity (Fig6C\&D), this is unsurprising, as we would not expect increased inhibitory tone from an exogenous ligand to affect neuronal connectivity. Overall, these results demonstrate that the changes in neuronal dynamics and activity with age can be effectively reduced by increased GABA signaling, further suggesting that the loss of inhibitory signaling with age contributes to early neuronal decline.

The UNC-2/CaV2 $\alpha$ channel has been directly tied to the removal of GABAergic synapses in Dorsal D-type motor neurons during development (Miller-Fleming et al., 2016). This process appears to be partially reliant upon calcium-mediated CED-4/Apaf-1 signaling. CED-4/Apaf-1 is a key mediator of the conserved apoptotic caspase pathway which has been shown to contribute to the removal of presynaptic domains in $C$. elegans (Meng et al., 2015) (Miller-Fleming et al., 2016), synapse elimination in higher order species (Ertürk et al., 2014) (Wang et al., 2014) (Li et al., 2010), and more substantial neuronal remodeling, such as regeneration following injury (Pinan-Lucarre et al., 2012) (Wang et al., 2019). We find that suppression of the caspase activator CED-4 reduces age-associated decline in neuron dynamics in a manner similar to that of unc-2 loss- 
of-function (If) mutation. Ced-4 mutation prevents the shift in spectral power toward higher frequencies in day 9 animals (Fig5A\&B) and also substantially reduces the loss of inhibitory signaling, helping to maintain the excitatory/inhibitory balance (Fig5C\&D). However, any effects on system organization are minimal (Fig5E). Our results thus far suggest that a pathway involving UNC-2/CaV2 $\alpha$ mediating calcium activation of caspases could play a non-apoptotic role in neuronal aging, resulting in progressive degradation of inhibitory signaling with age. Further investigations will be needed to elucidate additional components and mechanisms of this pathway.

Finally, we found that many aspects of neuronal dynamics in C. elegans are preserved in long-lived animals but exhibited premature decline in a neurodegenerative model. daf-2 is a well-studied model for longevity in $C$. elegans that acts through the silencing of the insulin signaling pathway. Importantly, daf-2 animals also display extended "healthspan" with preserved behavioral traits and mobility later in life (Podshivalova et al., 2017). Correspondingly, we find that the frequency dynamics of neuronal activity and system-wide dynamics are partially preserved in daf-2 animals, while neuronal correlativity is not, demonstrating that specific aspects of neuronal aging are mitigated by silencing of the insulin/ IGF-1 pathway. In contrast, recent work in C. elegans has shown that loss of sel-12 leads to disruptions in calcium homeostasis, eliciting mitochondrial dysfunction and neurodegeneration during early life (Sarasija et al., 2018). Importantly, sel-12 encodes the C. elegans orthologue to mammalian presenilin PSEN2, mutations of which are directly associated with familial early onset Alzheimer's disease (Cruts and Van Broeckhoven, 1998). We find that sel-12 animals prematurely display many attributes of age-associated neuronal dynamics, including loss of inhibitory signaling, higher dynamic frequencies, and the disruption of system-wide organization and continuity. Interestingly, they also display a decrease in strongly correlated neuron pairs that we do not observe in normal aging, suggesting changes in neuron-connectivity that are unique to sel-12 neurodegeneration. Our findings highlight the similarities and differences in neuronal dynamics between normal aging and this neurodegenerative context.

In this study, we have performed the first comprehensive assessment of activity dynamics across a large swath of the aging $C$. elegans nervous system. Our results highlight the conserved similarities with neuronal aging in mammals and further $C$. elegans as a robust model for the study of functional neuronal decline in aging and neurodegenerative diseases. Our unique measurements allow us to link a breakdown in system-wide organization and dynamics to changes in individual neuron activity and signaling. We identify a loss of inhibitory signaling as a key element of early aging resulting in disruption of excitatory/inhibitory neuronal homeostasis. These changes appear to involve UNC-2/CaV2 $\alpha$ and CED-4/Apaf-1 signaling and are mitigated by application of a $\mathrm{GABA}_{\mathrm{A}}$ agonist. With this study, we determine how changes in neuronal signaling and activity drive age-related decline in nervous system dynamics and begin to identify the underlying cellular processes. Our work provides a powerful system for the understanding of the genetic and circuit-level underpinnings of normal aging and neuropathological states.

\section{MATERIALS AND METHODS}

\section{C. elegans Strains and Maintenance}

Hermaphrodite $C$. elegans were maintained at $20^{\circ} \mathrm{C}$ on nematode growth medium (NGM)-agarose plates coated with food-source Escherichia coli strain OP50. Experiments were performed on Days 1, 3, 6, 9, and 12 of nematode adulthood. Age-synchronization was accomplished via timed egg-lays, during which gravid adults were allowed to lay eggs on NGM plates for a period of $2 \mathrm{~h}$, before removal. Adult worms were regularly transferred to fresh plates, as needed, to prevent starvation and to separate the aging adults from their progeny, except when aged on plates containing 50 uM 5-Fluoro-2'-deoxyuridine (FUdR) specifically for Spontaneous Reversal Rate behavioral experiments. Confocal microscopy and associated behavioral experiments were performed using the transgenic strain QW1574 (lite-1[ce314], zfis146[nmr-

$1::$ NLSwCherry::SL2::GCaMP6s, lim-4(-3328-2174)::NLSwCherry::SL2::GCaMP6s, lgc-55(-120-

773)::NLSwCherry::SL2::GCaMP6s, npr-9::NLSwCherry::SL2::GCaMP6s] \#18.9 [from zfex696]), which 
expresses the nuclear-localized red fluorescent protein NLSwCherry and cytoplasmic calcium-sensitive green fluorescent protein GCaMP6s in eight pairs of premotor interneurons (AVA, AVB, AVD, AVE, AIB, RIM, RIV, and PVC) in an otherwise unmodified wild-type N2 background (Awal et al., 2018). When indicated, behavioral and confocal experiments were performed using QW1574 worms that had been crossed with the long-lived strain CB1370 (daf-2[e1370]), which harbors a suppressive mutation within the daf-2 insulin/ insulin-like growth factor 1 receptor orthologue. Light-sheet microscopy and associated behavioral experiments were performed using the transgenic strain QW1217 (zfls124[Prgef-1::GCaMP6s]; otls355[Prab-3::NLS::tagRFP]), which panneuronally expresses nuclear-localized tagRFP and cytoplasmic GCaMP6s. When indicated, light-sheet microscopy and associated behavioral experiments were performed using QW1217 worms that had been crossed with strain CB1370 (daf-2[e1370]), CB55 (unc-2[e55]), QW37 (unc-2[zf35]), MT2547 (ced-4[n1162]) or AN87 (sel-12[ty11]). CB55 and QW37 harbor loss- and gain-of-function mutations, respectively, within the CaV2 channel $\alpha 1$ subunit UNC-2. MT2547 harbors a suppressive mutation within CED-4, a key caspase activator within the conserved apoptotic cell-death pathway and homolog to Apaf-1. AN87 harbors a mutation which introduces a premature stop codon within the PSEN2 (presenilin 2) orthologue SEL-12. The following strains and crosses were generated in the laboratory of Dr. Mark Alkema (University of Massachusetts Medical School, Worcester, Massachusetts): QW1574, QW1217, QW1319 (QW1217; CB55), and QW1348 (QW1217; QW37). The following strains were obtained from the Caenorhabditis Genetics Center (University of Minnesota, Minneapolis, Minnesota): CB55 (unc-2[e55]), MT2547 (ced-4[n1162]), CB1370 (daf-2[e1370]), and AN87 (sel-12[ty11]).

\section{Behavior}

All C. elegans behavior was assessed in a food-free environment. Spontaneous Reversal Rate: Immediately before transfer to food-free NGM-agarose plates, worms were washed $3 \mathrm{x}$ in $1 \times$ S-Basal solution (100 mM $\mathrm{NaCl}, 50 \mathrm{mM} \mathrm{KPO}_{4}$ buffer, $5 \mu \mathrm{g} / \mathrm{ml}$ cholesterol) to remove bacteria. Animals were then left undisturbed for 10 minutes on clean plates, before assessment. Crawling behavior was video recorded and subsequently analyzed using ImageJ (Rueden et al., 2017). The spontaneous reversal rates of individual animals were determined by counting the number of spontaneous reversals initiated during timeframes ranging from 2 to 10 minutes. Anterior Touch Responsiveness: To facilitate the removal of bacteria, worms were moved to fresh NGM-agarose plates (without bacteria) and allowed to crawl around for $5 \mathrm{~min}$. This was repeated once more, before animals were transferred to a third and final plate, where they were left undisturbed for 10 min prior to behavioral assessments. Individual animals, locomoting in a forward direction, were stroked with an eyelash across the anterior portion of their bodies a total of 5 times, with a recovery period of at least 5 min between stimuli. Animals were scored as responsive or non-responsive to a given stimulus, if a movement reversal was elicited or not, respectively. Worm populations were blinded to experimenters performing behavioral measurements.

\section{Confocal Microscopy}

Confocal fluorescence microscopy was performed as previously described (Wirak et al., 2020) to capture spontaneous in vivo activity of the premotor interneuron AVA. In short, worms were briefly paralyzed in $5 \mathrm{mM}$ tetramisole immediately prior to encasement in a transparent and permeable polyethylene hydrogel (Burnett et al., 2018). AVA was then imaged for 10 minutes at a rate of 4 images/second and its activity dynamics captured via calcium indicator GCaMP6s fluorescence. Nuclear-localized RFP fluorescence was captured in parallel, to facilitate downstream image registration.

\section{Light-sheet Microscopy}

Light-sheet microscopy and imaging preparation were performed as previously described (Awal et al., 2020), to capture spontaneous in vivo neural activity within the animal's entire head-region at single-cell resolution. Minor changes in protocol include the covalent attachment of the crosslinked polyethylene hydrogel to a silanized glass coverslip (prepared as described (Burnett et al., 2018)), instead of hydrogel-coverslip attachment via cyanoacrylate ester. Briefly, following hydrogel encapsulation, worms were placed in a Petri 
dish and immersed in $1 \times$ S-Basal solution $\left(100 \mathrm{mM} \mathrm{NaCl}, 50 \mathrm{mM} \mathrm{KPO}_{4}\right.$ buffer, $5 \mu \mathrm{g} / \mathrm{ml}$ cholesterol) with $5 \mathrm{mM}$ tetramisole. Imaging was performed using a Dual Inverted Selective Plane Illumination (diSPIM) fluorescence microscope (Applied Scientific Instrumentation, USA) with water immersion 0.8 NA 40x objectives (Nikon USA, Melville, NY). Each animal was imaged for 10 minutes at a rate of 2 volumes/second (voxel size $0.1625 \mathrm{x}$ $0.1625 \times 1 \mu \mathrm{m})$, capturing both nuclear-RFP and cytoplasmic-GCaMP6s fluorescence. Custom Python and MATLAB (Mathworks, USA) scripts were employed during postprocessing to track 120 RFP-labelled nuclei per animal in three dimensions and extract GCaMP6s signals from the surrounding somas. When indicated, the 1 $\times$ S-Basal solution in which the animals were immersed was supplemented with $1 \mathrm{mM}$ muscimol.

\section{Data Analysis}

All GCaMP6s fluorescence intensity traces were normalized $\Delta F / F_{0}$ (i.e. the change in fluorescence above baseline, divided by that baseline). AVA Activity: From the normalized AVA activity traces, calcium transient onsets (i.e. on transitions) and offsets (i.e. off transitions) were manually identified by clear rises and falls in GCaMP6s fluorescence intensity. As previously described (Wirak et al., 2020), nonlinear fitting of hyperbolic tangent functions to these on and off transitions facilitated the unbiased analysis of activity state transition properties and eliminated noise. To determine the Rise Time of a given on transition, its fitted function was used to calculate the time required to rise from $5 \%$ of the maximum fluorescence above baseline to $95 \%$. Off transition Fall Times (i.e. the time required to fall from $95 \%$ to $5 \%$ of the max fluorescence above baseline) were similarly determined. Average on and off transition plots were generated by aligning individual traces such that the calculated $5 \% \max \left(\Delta F / F_{0}\right)$ and $95 \% \max \left(\Delta F / F_{0}\right)$ positions, respectively, aligned at the same frame, prior to averaging. To determine the proportion of time that AVA was active versus inactive, its duty ratio was calculated. This was done by summing the calcium transient durations for a given GCaMP6s trace and dividing by the total length of the trace (i.e. 10 minutes). The duration of a given calcium transient was defined as the time difference between the first frame of its onset and the first frame of its offset. Multi-neuron

Activity: Each imaging condition consists of 10-20 animals, with activity traces pulled from 120 neurons per animal. Principal component analysis was performed on the normalized fluorescence traces $\left(\triangle F / F_{0}\right)$ and $P C A$ figures generated by plotting the first three principle components on a 3D graph to generate a trajectory over time. Smoothness of these trajectories over time was assessed by calculating the instantaneous change in direction at each time point, i.e. the absolute angular difference between the tangential direction over the past three seconds and that of the future three seconds. This calculation amounts to a discrete time derivative of the tangential angle describing the trajectory defined within an intrinsic co-ordinate system and is widely used in analyzing 3-dimensional trajectories. Probability histograms for individual animals (trials) or across all trials of a specific condition where generated by pooling the measurements of angular change from all timepoints within that/those trial(s). Prior to further analyses, time differentials were calculated from normalized activity arrays using total-variation regularization (Chartrand, 2011). To calculate mean power spectral densities (PSDs) for each condition (Fig3A), PSDs were generated for each worm's 120-neuron activity array, by Fourier transformation, and then averaged. To account for potential age-dependent changes in GCaMP6s expression and reporter function, these average PSDs were then normalized such that the total power of each is equal to 1. Average PSDs are alternatively displayed as cumulative PSD plots (Fig3A, inset), displaying the proportion of power residing below a given frequency. Relative shifts in PSD power were quantified by calculating for each condition the average $40 \%$ spectral edge (i.e. the frequency below which resides $40 \%$ of the total spectral power) (Fig3B). This was done by averaging each worm's $40 \%$ spectral edge. This particular spectral edge was chosen because it demonstrated the most prominent age-dependent shift in wild-type animals. Neuronneuron correlativity was further determined for each possible neuron pair among the 40 most dynamically active neurons (i.e. those having the greatest standard deviation in neuronal signal) within a given worm's 120neuron activity array. This was calculated between the GCaMP6s fluorescence time differentials of the two neurons. The 40 most dynamically active neurons were specifically examined in order to focus on those exhibiting highly dynamic, as opposed to static, activity. Neuron pair correlativity probability histograms (Fig3C) were generated for each condition by pooling all neuron-neuron correlativity values across all animals. To determine the proportion of neuron pairs exhibiting strong negative correlativity, the Negative Correlation Proportion (i.e. the proportion of correlativity values less than -0.2) (Fig3D) was calculated for each animal and averages generated for each condition. Likewise, to determine the proportion of neuron pairs exhibiting strong 
positive correlativity, the Positive Correlation Proportion (i.e. the proportion of correlativity values greater than 0.2) (Fig3E) was calculated for each animal and averages generated for each condition.

\section{Statistical Methods}

One-way ANOVA analyses were initially performed for each metric, across all conditions. Metrics were then compared across all conditions, between figures, with post hoc Sidak multiple comparisons tests. Wild-type data displayed in the figures is the same data set and is displayed versus the relevant data for that figure.

ACKNOWLEDGEMENTS: We thank Jennifer Luebke, Monica Driscoll, Harrison Gabel and all members of the Gabel lab for critical discussions related to the paper. We thank Vickery Trinkaus-Randall, Ph.D., director of the Boston University School of Medicine Confocal Microscopy Core, where confocal microscopy experiments were performed (Boston University School of Medicine, Boston, MA). Many strains used in this paper were obtained from the Caenorhabditis Genetics Center (University of Minnesota, Minneapolis, Minnesota). Funding was provided by the National Institute for General Medical Sciences (grant R01 GM121457 to C.W.C. and C.V.G. and grant T32 GM008541 to G.S.W.) and the National Institute for Neurological Disorders and Stroke (grant R01 NS107475 to M.J.A.).

AUTHOR CONTRIBUTIONS: G.S.W. and C.V.G. designed experiments. J.F. and M.J.A. generated C. elegans strains and crosses specified in the Methods. G.S.W. performed experiments. G.S.W., C.W.C., and C.V.G. aided in the analysis of data. G.S.W. and C.V.G. wrote the manuscript with input from all authors.

COMPETING INTERESTS: The authors declare no competing interests. 
Age-related loss of inhibitory signaling in C. elegans.

\section{LEGENDS (FIGURES)}

Figure 1: Activity dynamics of the AVA command interneuron with age. (A) Travel paths of two and three C. elegans, respectively, on day 1 and day 9 of adulthood (over 30 seconds). (B) Spontaneous reversal rates of adult worms of various ages, normalized to day 1 frequency. (For days 1, 3, 6, and 9, $n=9,18,22$, and 14 animals, respectively). (C) Anterior touch responsiveness in adult worms of various ages. Responsiveness denotes how often an eyelash drawn across the anterior portion of an animal's body elicited a reversal (see Methods). (For days 1, 3, 6, 9, and 12, $\mathrm{n}=20,44,53,53$, and 33 animals, respectively). (D) Transgenic expression of the calcium-sensitive green fluorescent protein GCaMP6s in the command interneuron AVA. (E) GCaMP calcium transients measured from the AVA interneuron acquired from animals at day 1 and 9 of adulthood. (F) Average AVA GCaMP fluorescence transient onsets and offsets in day 1 and 9 worms (shaded areas delineate the standard error of the mean). (For day 1 and 9 onsets, $n=37$ and 38 traces from 20 and 16 animals, respectively. For day 1 and 9 offsets, $n=33$ and 32 from 19 and 14 animals, respectively). (G, H) The average rise time (i.e. transient time from a "ON" transition from $5 \%$ to $95 \%$ of maximum fluorescence) and average fall time (95\% to 5\%) Of AVA GCaMP fluorescence transients at various ages. (For day 1, 3, 6, 9, and 12 onsets, $n=37,34,36,38$, and 43 traces from $20,13,13,16$, and 14 animals, respectively. For day $1,3,6$, 9 , and 12 offsets, $n=33,24,28,32$, and 29 traces from 20,11,11, 14, and 13 animals, respectively. (I) Duty ratio of AVA fluorescence, defined as the proportion of time that AVA is active versus inactive (see Methods). (For days 1, 3, 6, 9, and 12, $\mathrm{n}=21,13,13,12$, and 16 animals, respectively). Error bars denote standard error of the mean. ${ }^{*} \mathrm{P}<0.05 ;{ }^{* *} \mathrm{P}<0.01 ;{ }^{* *} \mathrm{P}<0.001$, Sidak post hoc test.

Figure 2. Breakdown of system dynamics and state organization with age. (A) Example traces of 120neuron GCaMP fluorescence measurements, captured in the head-region of a day 1 young adult and a day 9 senescent worm (blue and red represent low and high fluorescence respectively). (B) Time correlation heatmaps derived of the fluorescence measurements in A. The relative distance in activity space is calculated between every pair of time points in the trial (see Methods) indicated by color (blue indicates zero, red indicates maximal distance). (C) 3-dimensional plots displaying the trajectories of the first three principal components derived from fluorescence measurements in A. Insets are probability histograms of the angular directional changes for those principal component trajectories (see Methods). (D) Aggregate probability histograms of the angular directional changes in principal component trajectories for all animals measured at various ages. (For $\mathrm{D}, \mathrm{n}=20,10,10,20,10$ animals for days 1, 3, 6, 9, and 12, respectively).

Figure 3. Neuron activity dynamics and correlation with age. (A) Mean power spectral densities (PSDs) derived from the fluorescence traces of individual neurons measured at various ages. Inset: cumulative PSD plots, display the proportion of power residing below a given frequency. (B) Mean $40 \%$ spectral edges across neuron PSDs at various ages, denoting the average frequency below which resides $40 \%$ of the total spectral power. (For $A$ and $B, n=120$ neurons per animal with 20 animals per condition). (C) Aggregate probability histograms of neuron-pair correlativity among the 40 most dynamically active neurons, in worms at day 1 and 9 of adulthood (see Methods). (D) The Negative Correlation Proportion, denoting the measured proportion of strongly negative neuron-pair correlativity values $(<-0.2)$. (E) The Positive Correlation Proportion, denoting the measured proportion of strongly positive neuron-pair correlativity values $(>0.2)$. (For $C-E, n=40$ neurons per animal with 20,10,10,20, and 10 animals for days $1,3,6,9$, and 12 , respectively). Error bars denote standard error of the mean. ${ }^{*} \mathrm{P}<0.05 ;{ }^{* *} \mathrm{P}<0.01 ;{ }^{* *} \mathrm{P}<0.001$, Sidak post hoc test.

Figure 4. Neuron activity dynamics of unc-2 mutant animals with age. (A) Spontaneous reversal rates of wild-type worms, unc-2(e55) loss-of-function (If) mutant worms, and unc-2(zf35) gain-of-function (gf) mutant worms on days 1 and 6 of adulthood. (On days 1 and $6, n=28$ and 26 for wild-type worms, 26 and 26 for unc2(If) worms, and 25 and 22 for unc-2(gf) worms, respectively). (B) Mean power spectral densities (PSDs) of the neuron GCaMP fluorescence in wild-type, unc-2(If), and unc-2(gf) worms on days 1 and 9 of adulthood. Insert: cumulative PSD plots. (C) Mean $40 \%$ spectral edges of the individual PSDs used to generate graphs in B. (For $\mathrm{B}$ and $\mathrm{C}, \mathrm{n}=120$ neurons per animal with 17-20 animals per condition). (D) Aggregate probability histograms, and $(E)$ the Negative Correlation Proportion, of neuron-pair correlativity among the 40 most dynamically active neurons, in wild-type, unc-2(If), and unc-2(gf) worms on days 1 and 9 of adulthood. (For D and E, n = 40 neurons per animal with 17-20 animals per condition). (F) Aggregate probability histograms of the angular 
directional changes in PCA trajectories for wild-type, unc-2(If), and unc-2(gf) worms on days 1 and 9 of adulthood. (Each PCA trajectory was generated with 120 neurons per animal with 17-20 animals per condition). Error bars denote standard error of the mean. ${ }^{*} P<0.05$; ${ }^{* *} P<0.01$; ${ }^{* * *} P<0.001$, Sidak post hoc test.

Figure 5. Ced-4 mutation diminishes age-associated changes in neuronal dynamics. (A) Mean power spectral densities (PSDs) of the neuron GCaMP fluorescence traces in wild-type and ced-4(n1162) loss-offunction worms on days 1 and 9 of adulthood. Insert: cumulative PSD plots (B) Mean 40\% spectral edges of the individual PSDs used to generate graphs in $A$. (For $A$ and $B, n=120$ neurons per animal with 18-20 animals per condition). (C) Aggregate probability histograms, and (D) the Negative Correlation Proportion, of neuron-pair correlativity among the 40 most dynamically active neurons, in wild-type and ced-4 worms on days 1 and 9 of adulthood. (For $C$ and D, $n=40$ neurons per animal with 18-20 animals per condition). (E) Aggregate probability histograms of the angular directional changes of PCA trajectories for wild-type and ced-4 worms on days 1 and 9 of adulthood. (Each PCA trajectory was generated with 120 neurons per animal with 18-20 animals per condition). Error bars denote standard error of the mean. ${ }^{*} \mathrm{P}<0.05 ;{ }^{* *} \mathrm{P}<0.01$; ${ }^{* * *} \mathrm{P}<$ 0.001 , Sidak post hoc test.

Figure 6. Acute muscimol application rescues several age-associated changes in neuron dynamics. (A) Mean power spectral densities (PSDs) of the neuron GCaMP fluorescence traces in untreated and muscimoltreated animals on days 1 and 9 of adulthood. Insert: cumulative PSD plots. (B) Mean 40\% spectral edges of the individual PSDs used to generate graphs in A. (For $A$ and $B, n=120$ neurons per animal with 17-20 animals per condition). (C) Aggregate probability histograms, and (D) the Negative Correlation Proportion, of neuron-pair correlativity among the 40 most dynamically active neurons, in untreated and muscimol-treated worms on days 1 and 9 of adulthood. (For $C$ and $D, n=40$ neurons per animal with 17-20 animals per condition). (E) Aggregate probability histograms of the angular directional changes of PCA trajectories in untreated and muscimol-treated worms on days 1 and 9 of adulthood. (Each PCA trajectory was generated with 120 neurons per animal with 17-20 animals per condition). Error bars denote standard error of the mean. ns $\mathrm{P}>0.05 ;{ }^{*} \mathrm{P}<0.05 ;{ }^{* *} \mathrm{P}<0.01$; ${ }^{* * *} \mathrm{P}<0.001$, Sidak post hoc test.

Figure 7. Effects of long-lived daf-2 mutation and neurodegenerative sel-12 mutation on age-associated changes in neuronal dynamics. (A\&C) Mean power spectral densities (PSDs) of the neuron GCaMP fluorescence traces in wild-type, daf-2(e1370) loss-of-function, and sel-12(ty11) truncation worms on days 1, 3, and 9 of adulthood. Insert: cumulative PSD plots (B\&D) Mean $40 \%$ spectral edges of the individual PSDs used to generate graphs in $A$. (For $A$ and $B, n=120$ neurons per animal with 18-20 animals per condition). (E\&G) Aggregate probability histograms, and (F\&H) the Negative Correlation Proportion of neuron-pair correlativity among the 40 most dynamically active head-region neurons, in wild-type, daf-2, and sel-12 worms on days 1 and 9 of adulthood. (For $C$ and $D, n=40$ neurons per animal with 18-20 animals per condition). (I\&J) Aggregate probability histograms of the angular directional changes of PCA trajectories for wild-type, daf-2, and sel-12 worms on days 1, 3, and 9 of adulthood. (Each PCA trajectory was generated with 120 neurons per animal with 18-20 animals per condition). Error bars denote standard error of the mean. ${ }^{*}<<0.05$; ${ }^{* *} P<0.01$; ${ }^{* * *} \mathrm{P}<0.001$, Sidak post hoc test. 


\section{LEGENDS (SUPPLEMENTAL FIGURES)}

Figure S1: AVA calcium transient frequency and duration. GCaMP calcium transient (A) frequency and (B) duration in the AVA interneuron. (For days 1, 3, 6, 9, and 12 onsets, $n=21,13,13$, 12, and 16 animals, respectively). Error bars denote standard error of the mean. ${ }^{*} \mathrm{P}<0.05$; ${ }^{* *} \mathrm{P}<0.01$; ${ }^{* *} \mathrm{P}<0.001$, Sidak post hoc test.

Figure S2: Multi-neuron activity from individual worms with age. From left to right column: Example traces of 120-neuron GCaMP fluorescence measurements, captured in the head-region of individual adult worms at various ages (blue and red represent low and high fluorescence respectively). Time correlation heatmaps derived of the fluorescence measurements in the 120-neuron GCaMP arrays, where the relative distance in activity space is calculated between every time point (see Statistical Methods) (blue represents two time points with very similar neuronal activity patterns while red indicates significantly different neuronal activity patterns). 3-dimensional plots displaying the trajectories of the first three principal components derived from fluorescence measurements in the 120-neuron GCaMP arrays. Probability histograms of the angular directional changes for those principal component trajectories (see Statistical Methods) (histograms highly skewed toward low angular changes reflect plots with smooth trajectories over time, whereas histograms with a broader distribution of angular changes reflect plots with erratic trajectories).

Figure S3. AVA activity dynamics in daf-2 mutant animals with age. (A) Spontaneous reversal rates of wild-type and daf-2 worms at day 1 and 6 of adulthood. (For wild-type days 1 and $6, n=28$ and 26 animals, respectively. For daf-2 days 1 and $6, n=38$ and 20 animals, respectively). (B) Average AVA GCaMP fluorescence transient onsets and offsets in day 1 and 9 daf-2(e1370) worms (shaded areas delineate the standard error of the mean). (For day 1 and 9 onsets, $n=12$ and 30 traces from 7 and 15 animals, respectively. For day 1 and 9 offsets, $n=15$ and 29 from 8 and 12 animals, respectively). (C, D) The average rise time (i.e. transient time from a "ON" transition from $5 \%$ to $95 \%$ of maximum fluorescence) and average fall time $(95 \%$ to $5 \%)$ Of AVA GCaMP fluorescence transients at various ages. (For wild-type day 1 and 9 onsets, $\mathrm{n}=37$ and 38 traces from 20 and 16 animals, respectively. For daf-2 day 1 and 9 onsets, $\mathrm{n}=12$ and 29 traces from 7 and 14 animals, respectively. For wild-type day 1 and 9 offsets, $n=33$ and 32 traces from 20 and 14 animals, respectively. For daf-2 day 1 and 9 offsets, $n=15$ and 29 traces from 8 and 12 animals, respectively. (E) Duty ratio of AVA fluorescence, defined as the proportion of time that AVA is active versus inactive (see Statistical Methods). (For wild-type day 1 and $9, \mathrm{n}=21$ and 12 animals, respectively. For daf-2 day 1 and $9, \mathrm{n}$ $=15$ and 15 animals, respectively). Error bars denote standard error of the mean. ${ }^{*} \mathrm{P}<0.05 ;{ }^{* *} \mathrm{P}<0.01 ;{ }^{* * *} \mathrm{P}$ $<0.001$, Sidak post hoc test. 
Age-related loss of inhibitory signaling in C. elegans.

\section{REFERENCES}

Awal, M.R., Austin, D., Florman, J., Alkema, M., Gabel, C. V., and Connor, C.W. (2018). Breakdown of Neural Function under Isoflurane Anesthesia. Anesthesiology. 129(4):733-743. doi: 10.1097/ALN.0000000000002342

Awal, M.R., Wirak, G.S., Gabel, C. V., and Connor, C.W. (2020). Collapse of global neuronal states in caenorhabditis elegans under isoflurane anesthesia. Anesthesiology. 133(1):133-144. doi: 10.1097/ALN.0000000000003304

Bagarinao, E., Watanabe, H., Maesawa, S., Mori, D., Hara, K., Kawabata, K., Yoneyama, N., Ohdake, R., Imai, K., Masuda, M., et al. (2019). Reorganization of brain networks and its association with general cognitive performance over the adult lifespan. Sci. Rep. 9(1):11352. doi: 10.1038/s41598-019-47922-x

Bolanowski, M.A., Russell, R.L., and Jacobson, L.A. (1981). Quantitative measures of aging in the nematode Caenorhabditis elegans. I. Population and longitudinal studies of two behavioral parameters. Mech. Ageing Dev. 15(3):279-95. doi: 10.1016/00476374(81)90136-6

Burnett, K., Edsinger, E., and Albrecht, D.R. (2018). Rapid and gentle hydrogel encapsulation of living organisms enables long-term microscopy over multiple hours. Commun. Biol. 1:73. doi: 10.1038/s42003-018-0079-6

Buzsáki, G., and Watson, B.O. (2012). Brain rhythms and neural syntax: Implications for efficient coding of cognitive content and neuropsychiatric disease. Dialogues Clin. Neurosci. 14(4):345-67. doi: 10.31887/DCNS.2012.14.4/gbuzsaki

Chalfie, M., Sulston, J.E., White, J.G., Southgate, E., Thomson, J.N., and Brenner, S. (1985). The neural circuit for touch sensitivity in Caenorhabditis elegans. J. Neurosci. 5(4):956-64. doi: 10.1523/JNEUROSCI.05-04-00956.1985

Chartrand, R. (2011). Numerical Differentiation of Noisy, Nonsmooth Data. ISRN Appl. Math. Vol. 2011, Article ID 164564,11 pages. doi: 10.5402/2011/164564

Cheng, C.H., and Lin, Y.Y. (2013). Aging-related decline in somatosensory inhibition of the human cerebral cortex. Exp. Brain Res. 226(1):145-52. doi: 10.1007/s00221-013-3420-9

Chokshi, T.V., Bazopoulou, D., and Chronis, N. (2010). An automated microfluidic platform for calcium imaging of chemosensory neurons in Caenorhabditis elegans. Lab Chip. 10(20):2758-63. doi: 10.1039/c004658b

Cook, S.J., Jarrell, T.A., Brittin, C.A., Wang, Y., Bloniarz, A.E., Yakovlev, M.A., Nguyen, K.C.Q., Tang, L.T.H., Bayer, E.A., Duerr, J.S., et al. (2019). Whole-animal connectomes of both Caenorhabditis elegans sexes. Nature. 571(7763):63-71. doi: $10.1038 / \mathrm{s} 41586-019-1352-7$

Croll, N.A. (1975). Behavioural Analysis of Nematode Movement. Adv. Parasitol. 13:71-122. doi: 10.1016/s0065-308x(08)60319-x

Croll, N.A., Smith, J.M., and Zuckerman, B.M. (1977). The aging process of the nematode caenorhabditis elegans in bacterial and axenic culture. Exp. Aging Res. 3(3):175-89. doi: 10.1080/03610737708257101

Croll, N.A. (1975). Components and patterns in the behaviour of the nematode Caenorhabditis elegans. J. Zool. 176(2):159-176. doi: 10.1111/j.1469-7998.1975.tb03191.x

Cruts, M., and Van Broeckhoven, C. (1998). Presenilin mutations in Alzheimer's disease. Hum. Mutat. 11(3):183-90. doi: 10.1002/(SICI)1098-1004(1998)11:3<183::AID-HUMU1>3.0.CO;2-J

David-Jürgens, M., and Dinse, H.R. (2010). Effects of aging on paired-pulse behavior of rat somatosensory cortical neurons. Cereb. Cortex. 20(5):1208-16. doi: 10.1093/cercor/bhp185

Draguhn, A., and Buzsáki, G. (2004). Neuronal Oscillations in Cortical Networks. Science. 304(5679):1926-9. doi: 10.1126/science. 1099745

Ertürk, A., Wang, Y., and Sheng, M. (2014). Local pruning of dendrites and spines by caspase-3-dependent and proteasome-limited mechanisms. J. Neurosci. 34(5):1672-88. doi: 10.1523/JNEUROSCI.3121-13.2014

Felkai, S., Ewbank, J.J., Lemieux, J., Labbé, J.C., Brown, G.G., and Hekimi, S. (1999). CLK-1 controls respiration, behavior and aging in the nematode Caenorhabditis elegans. EMBO J. 18(7):1783-92. doi: 10.1093/emboj/18.7.1783

Fritschy, J.M. (2008). Epilepsy, E/I balance and GABAA receptor plasticity. Front. Mol. Neurosci. 1:5. doi: 10.3389/neuro.02.005.2008

Glenn, C.F., Chow, D.K., David, L., Cooke, C.A., Gami, M.S., Iser, W.B., Hanselman, K.B., Goldberg, I.G., and Wolkow, C.A. (2004). Behavioral deficits during early stages of aging in Caenorhabditis elegans result from locomotory deficits possibly linked to muscle frailty. Journals Gerontol. - Ser. A Biol. Sci. Med. Sci. 59(12):1251-60. doi: 10.1093/gerona/59.12.1251

Haithcock, E., Dayani, Y., Neufeld, E., Zahand, A.J., Feinstein, N., Mattout, A., Gruenbaum, Y., and Liu, J. (2005). Age-related changes of nuclear architecture in Caenorhabditis elegans. Proc. Natl. Acad. Sci. U. S. A. 102(46):16690-5. doi: 10.1073/pnas.0506955102

Herndon, L.A., Schmeissner, P.J., Dudaronek, J.M., Brown, P.A., Listner, K.M., Sakano, Y., Paupard, M.C., Hall, D.H., and Driscoll, M. (2002). Stochastic and genetic factors influence tissue-specific decline in ageing C. Elegans. Nature. 419(6909):808-14. doi: 10.1038/nature01135

Huang, T.T., Matsuyama, H.J., Tsukada, Y., Singhvi, A., Syu, R.T., Lu, Y., Shaham, S., Mori, I., and Pan, C.L. (2020). Agedependent changes in response property and morphology of a thermosensory neuron and thermotaxis behavior in Caenorhabditis elegans. Aging Cell. 19(5):e13146. doi: 10.1111/acel.13146

Huang, Y.C., Pirri, J.K., Rayes, D., Gao, S., Mulcahy, B., Grant, J., Saheki, Y., Francis, M.M., Zhen, M., and Alkema, M.J. (2019). Gain-of-function mutations in the UNC-2/CaV2 $\alpha$ channel lead to excitation-dominant synaptic transmission in C. elegans. Elife. 8:e45905. doi: 10.7554/eLife.45905 
Isaacson, J.S., and Scanziani, M. (2011). How inhibition shapes cortical activity. Neuron. 72(2):231-43. doi: 10.1016/j.neuron.2011.09.027

Kato, S., Kaplan, H.S., Schrödel, T., Skora, S., Lindsay, T.H., Yemini, E., Lockery, S., and Zimmer, M. (2015). Global Brain Dynamics Embed the Motor Command Sequence of Caenorhabditis elegans. Cell. 163(3):656-69. doi: 10.1016/j.cell.2015.09.034

Kauffman, A.L., Ashraf, J.M., orces-Zimmerman, M.R., Landis, J.N., and Murphy, C.T. (2010). Insulin signaling and dietary restriction differentially influence the decline of learning and memory with age. PLoS Biol. 8(5):e1000372. doi: 10.1371/journal.pbio.1000372

Kawano, T., Po, M.D., Gao, S., Leung, G., Ryu, W.S., and Zhen, M. (2011). An imbalancing act: Gap junctions reduce the backward motor circuit activity to bias C. elegans for forward locomotion. Neuron. 72(4):572-86. doi: 10.1016/j.neuron.2011.09.005

Kenyon, C., Chang, J., Gensch, E., Rudner, A., and Tabtiang, R. (1993). A C. elegans mutant that lives twice as long as wild type. Nature. 366(6454):461-4. doi: 10.1038/366461a0

Leinwand, S.G., Yang, C.J., Bazopoulou, D., Chronis, N., Srinivasan, J., and Chalasani, S.H. (2015). Circuit mechanisms encoding odors and driving aging-associated behavioral declines in Caenorhabditis elegans. Elife. 4:e10181. doi: 10.7554/eLife.10181

Li, Z., Jo, J., Jia, J.M., Lo, S.C., Whitcomb, D.J., Jiao, S., Cho, K., and Sheng, M. (2010). Caspase-3 activation via mitochondria is required for long-term depression and AMPA receptor internalization. Cell. 141(5):859-71. doi: 10.1016/j.cell.2010.03.053

Liu, J., Zhang, B., Lei, H., Feng, Z., Liu, J., Hsu, A.L., and Xu, X.Z.S. (2013). Functional aging in the nervous system contributes to age-dependent motor activity decline in C. elegans. Cell Metab. 18(3):392-402. doi: 10.1016/j.cmet.2013.08.007

Mack, H.I.D., Heimbucher, T., and Murphy, C.T. (2018). The nematode Caenorhabditis elegans as a model for aging research. Drug Discov. Today Dis. Model. 27:3-13. doi: 10.1016/j.ddmod.2018.11.001

Mattson, M.P., and Arumugam, T. V. (2018). Hallmarks of Brain Aging: Adaptive and Pathological Modification by Metabolic States. Cell Metab. 27(6):1176-1199. doi: 10.1016/j.cmet.2018.05.011

McQuail, J.A., Frazier, C.J., and Bizon, J.L. (2015). Molecular aspects of age-related cognitive decline: The role of GABA signaling. Trends Mol. Med. 21(7):450-60. doi: 10.1016/j.molmed.2015.05.002

Meng, L., Mulcahy, B., Cook, S.J., Neubauer, M., Wan, A., Jin, Y., and Yan, D. (2015). The Cell Death Pathway Regulates Synapse Elimination through Cleavage of Gelsolin in Caenorhabditis elegans Neurons. Cell Rep. 11(11):1737-48. doi: 10.1016/j.celrep.2015.05.031

Miller-Fleming, T.W., Petersen, S.C., Manning, L., Matthewman, C., Gornet, M., Beers, A., Hori, S., Mitani, S., Bianchi, L., Richmond, J., et al. (2016). The DEG/ENaC cation channel protein UNC-8 drives activity-dependent synapse removal in remodeling GABAergic neurons. Elife. 5:e14599. doi: 10.7554/eLife.14599

Morrison, J.H., and Baxter, M.G. (2012). The ageing cortical synapse: Hallmarks and implications for cognitive decline. Nat. Rev. Neurosci. 13(4):240-50. doi: 10.1038/nrn3200

Mulcahy, B., Holden-Dye, L., and O'Connor, V. (2013). Pharmacological assays reveal age-related changes in synaptic transmission at the Caenorhabditis elegans neuromuscular junction that are modified by reduced insulin signalling. J. Exp. Biol. 216(Pt 3):492-501. doi: $10.1242 /$ jeb.068734

Nguyen, J.P., Shipley, F.B., Linder, A.N., Plummer, G.S., Liu, M., Setru, S.U., Shaevitz, J.W., and Leifer, A.M. (2016). Whole-brain calcium imaging with cellular resolution in freely behaving Caenorhabditis elegans. Proc. Natl. Acad. Sci. U. S. A. 113(8):E1074-81. doi: 10.1073/pnas. 1507110112

Nichols, A.L.A., Eichler, T., Latham, R., and Zimmer, M. (2017). A global brain state underlies C. Elegans sleep behavior. Science. 356(6344):eaam6851. doi: 10.1126/science.aam6851

Pan, C.L., Peng, C.Y., Chen, C.H., and McIntire, S. (2011). Genetic analysis of age-dependent defects of the Caenorhabditis elegans touch receptor neurons. Proc. Natl. Acad. Sci. U. S. A. 108(22):9274-9. doi: 10.1073/pnas. 1011711108

Peters, A., Sethares, C., and Luebke, J.I. (2008). Synapses are lost during aging in the primate prefrontal cortex. Neuroscience. 152(4):970-81. doi: 10.1016/j.neuroscience.2007.07.014

Pierce-Shimomura, J.T., Morse, T.M., and Lockery, S.R. (1999). The fundamental role of pirouettes in Caenorhabditis elegans chemotaxis. J. Neurosci. 19(21):9557-69. doi: 10.1523/JNEUROSCI.19-21-09557.1999

Pinan-Lucarre, B., Gabel, C. V., Reina, C.P., Hulme, S.E., Shevkoplyas, S.S., Slone, R.D., Xue, J., Qiao, Y., Weisberg, S., Roodhouse, K., et al. (2012). The core apoptotic executioner proteins CED-3 and CED-4 promote initiation of neuronal regeneration in caenorhabditis elegans. PLoS Biol. 10(5):e1001331. doi: 10.1371/journal.pbio.1001331

Podshivalova, K., Kerr, R.A., and Kenyon, C. (2017). How a Mutation that Slows Aging Can Also Disproportionately Extend End-ofLife Decrepitude. Cell Rep. 19(3):441-450. doi: 10.1016/j.celrep.2017.03.062

Potier, B., Jouvenceau, A., Epelbaum, J., and Dutar, P. (2006). Age-related alterations of GABAergic input to CA1 Pyramidal neurons and its control by nicotinic acetylcholine receptors in rat hippocampus. Neuroscience. 142(1):187-201. doi: 10.1016/j.neuroscience.2006.06.040

Richardson, B.D., Ling, L.L., Uteshev, V. V., and Caspary, D.M. (2013). Reduced GABAA receptor-mediated tonic inhibition in aged rat auditory thalamus. J. Neurosci. 33(3):1218-27a. doi: 10.1523/JNEUROSCI.3277-12.2013

Rozycka, A., and Liguz-Lecznar, M. (2017). The space where aging acts: focus on the GABAergic synapse. Aging Cell. 16(4):634643. doi: 10.1111/acel.12605

Rueden, C.T., Schindelin, J., Hiner, M.C., DeZonia, B.E., Walter, A.E., Arena, E.T., and Eliceiri, K.W. (2017). ImageJ2: ImageJ for 
bioRxiv preprint doi: https://doi.org/10.1101/2021.07.07.451497; this version posted July 8, 2021. The copyright holder for this preprint (which was not certified by peer review) is the author/funder. All rights reserved. No reuse allowed without permission.

Age-related loss of inhibitory signaling in C. elegans.

the next generation of scientific image data. BMC Bioinformatics. 18(1):529. doi: 10.1186/s12859-017-1934-z

Sala-Llonch, R., Bartrés-Faz, D., and Junqué, C. (2015). Reorganization of brain networks in aging: a review of functional connectivity studies. Front. Psychol. 6:663. doi: 10.3389/fpsyg.2015.00663

Sarasija, S., Laboy, J.T., Ashkavand, Z., Bonner, J., Tang, Y., and Norman, K.R. (2018). Presenilin mutations deregulate mitochondrial $\mathrm{Ca} 2+$ homeostasis and metabolic activity causing neurodegeneration in Caenorhabditis elegans. Elife. 7:e33052. doi: $10.7554 /$ eLife. 33052

Schmidt, S., Redecker, C., Bruehl, C., and Witte, O.W. (2010). Age-related decline of functional inhibition in rat cortex. Neurobiol. Aging. 31(3):504-11. doi: 10.1016/j.neurobiolaging.2008.04.006

Tank, E.M.H., Rodgers, K.E., and Kenyon, C. (2011). Spontaneous age-related neurite branching in Caenorhabditis elegans. J. Neurosci. 31(25):9279-88. doi: 10.1523/JNEUROSCI.6606-10.2011

Toth, M.L., Melentijevic, I., Shah, L., Bhatia, A., Lu, K., Talwar, A., Naji, H., Ibanez-Ventoso, C., Ghose, P., Jevince, A., et al. (2012). Neurite sprouting and synapse deterioration in the aging Caenorhabditis elegans nervous system. J. Neurosci. 32(26):8778-90. doi: 10.1523/JNEUROSCI.1494-11.2012

Turrigiano, G.G., and Nelson, S.B. (2004). Homeostatic plasticity in the developing nervous system. Nat. Rev. Neurosci. 5(2):97-107. doi: $10.1038 / \mathrm{nrn} 1327$

Varangis, E., Habeck, C.G., Razlighi, Q.R., and Stern, Y. (2019). The Effect of Aging on Resting State Connectivity of Predefined Networks in the Brain. Front. Aging Neurosci. 11:234. doi: 10.3389/fnagi.2019.00234

Vayndorf, E.M., Scerbak, C., Hunter, S., Neuswanger, J.R., Toth, M., Parker, J.A., Neri, C., Driscoll, M., and Taylor, B.E. (2016). Morphological remodeling of C. elegans neurons during aging is modified by compromised protein homeostasis. Npj Aging Mech. Dis. 2:16001. doi: 10.1038/npjamd.2016.1

Vecchia, D., and Pietrobon, D. (2012). Migraine: A disorder of brain excitatory-inhibitory balance? Trends Neurosci. 35(8):507-20. doi: $10.1016 /$ j.tins.2012.04.007

Venkatachalam, V., Ji, N., Wang, X., Clark, C., Mitchell, J.K., Klein, M., Tabone, C.J., Florman, J., Ji, H., Greenwood, J., et al. (2016). Pan-neuronal imaging in roaming Caenorhabditis elegans. Proc. Natl. Acad. Sci. U. S. A. 113(8):E1082-8. doi: 10.1073/pnas. 1507109113

Vico Varela, E., Etter, G., and Williams, S. (2019). Excitatory-inhibitory imbalance in Alzheimer's disease and therapeutic significance. Neurobiol. Dis. 127:605-615. doi: 10.1016/j.nbd.2019.04.010

Wang, G., Sun, L., Reina, C.P., Song, I., Gabel, C. V., and M., D. CED-4 CARD domain residues can modulate non-apoptotic neuronal regeneration functions independently from apoptosis. Sci. Rep. 9(1):13315. doi: 10.1038/s41598-019-49633-9

Wang, J.Y., Chen, F., Fu, X.Q., Ding, C.S., Zhou, L., Zhang, X.H., and Luo, Z.G. (2014). Caspase-3 cleavage of dishevelled induces elimination of postsynaptic structures. Dev. Cell. 28(6):670-84. doi: 10.1016/j.devcel.2014.02.009

Wirak, G.S., Gabel, C. V., and Connor, C.W. (2020). Isoflurane Exposure in Juvenile Caenorhabditis elegans Causes Persistent Changes in Neuron Dynamics. Anesthesiology. 133(3):569-582. doi: 10.1097/ALN.0000000000003335

Zullo, J.M., Drake, D., Aron, L., O’Hern, P., Dhamne, S.C., Davidsohn, N., Mao, C.A., Klein, W.H., Rotenberg, A., Bennett, D.A., et al. (2019). Regulation of lifespan by neural excitation and REST. Nature. 574(7778):359-364. doi: 10.1038/s41586-019-1647-8 
Figure 1

a.

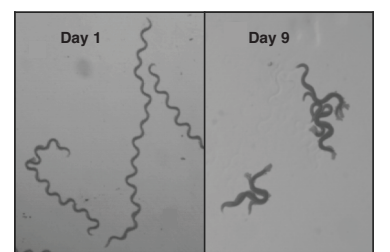

b.

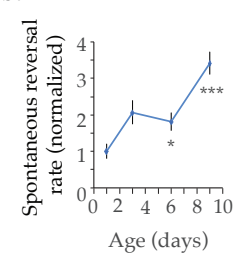

c.

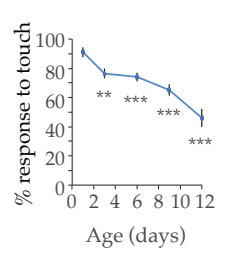

d.
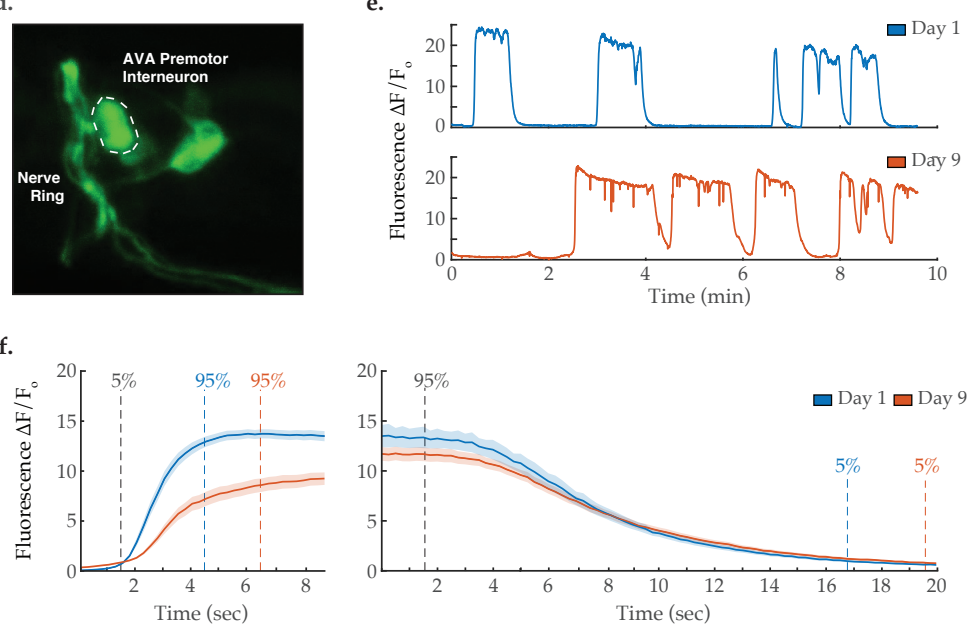

g.

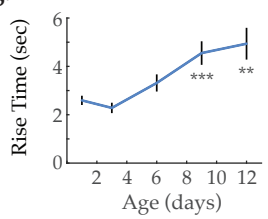

h.

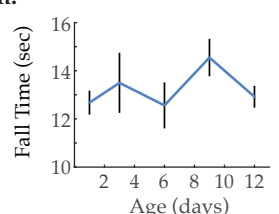

i.

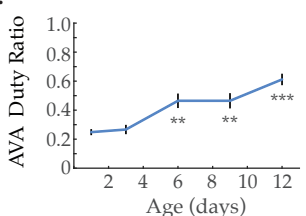


bioRxiv preprint doi: https://doi.org/10.1101/2021.07.07.451497; this version posted July 8, 2021. The copyright holder for this preprint (which was not certified by peer review) is the author/funder. All rights reserved. No reuse allowed without permission.

Figure 2

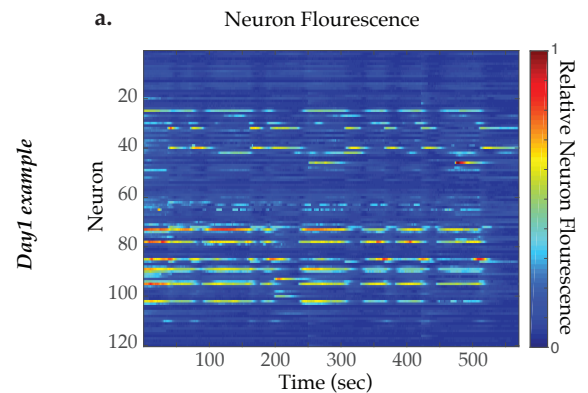

b. Time Correlation of System Activity
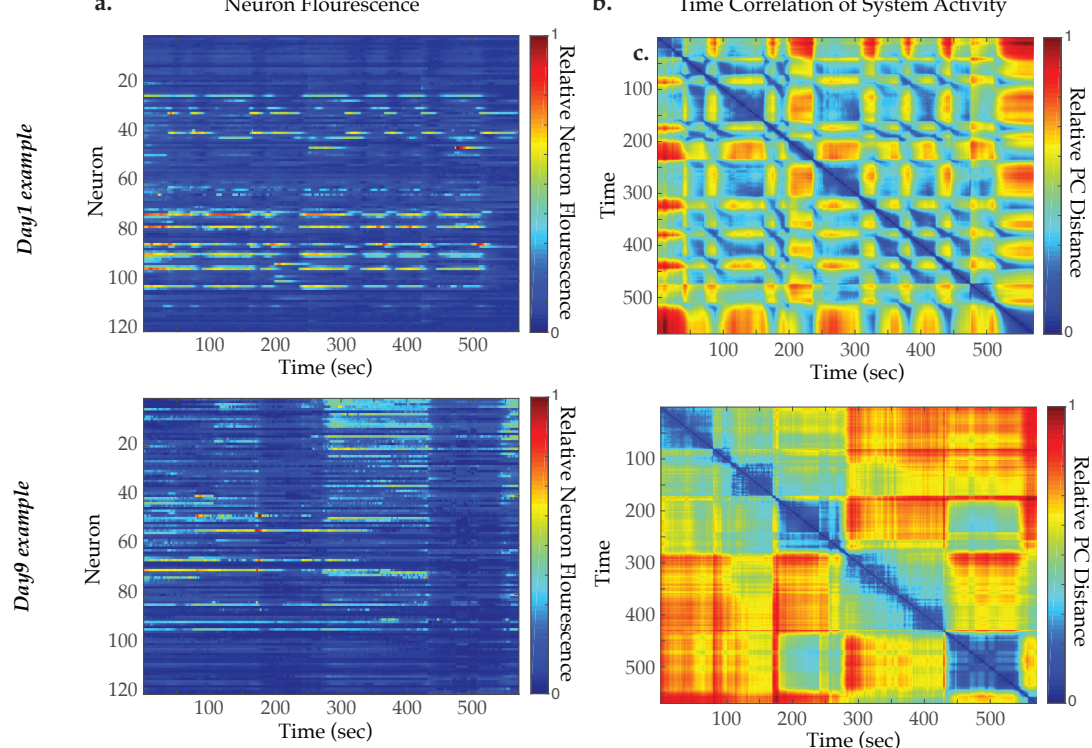

d.

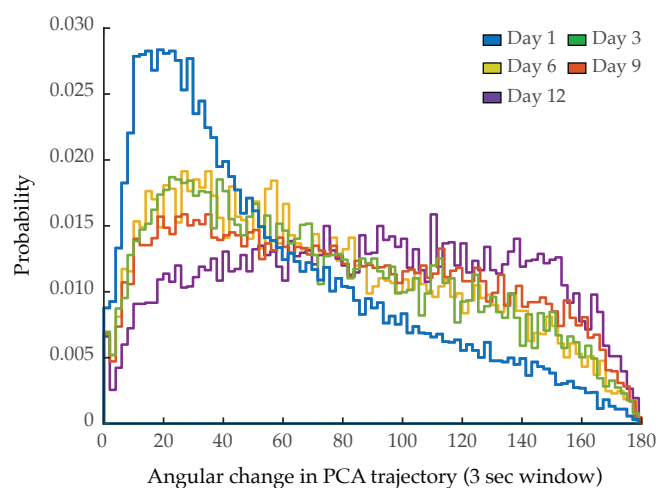


bioRxiv preprint doi: https://doi.org/10.1101/2021.07.07.451497; this version posted July 8, 2021. The copyright holder for this preprint (which was not certified by peer review) is the author/funder. All rights reserved. No reuse allowed without permission.

Figure 3
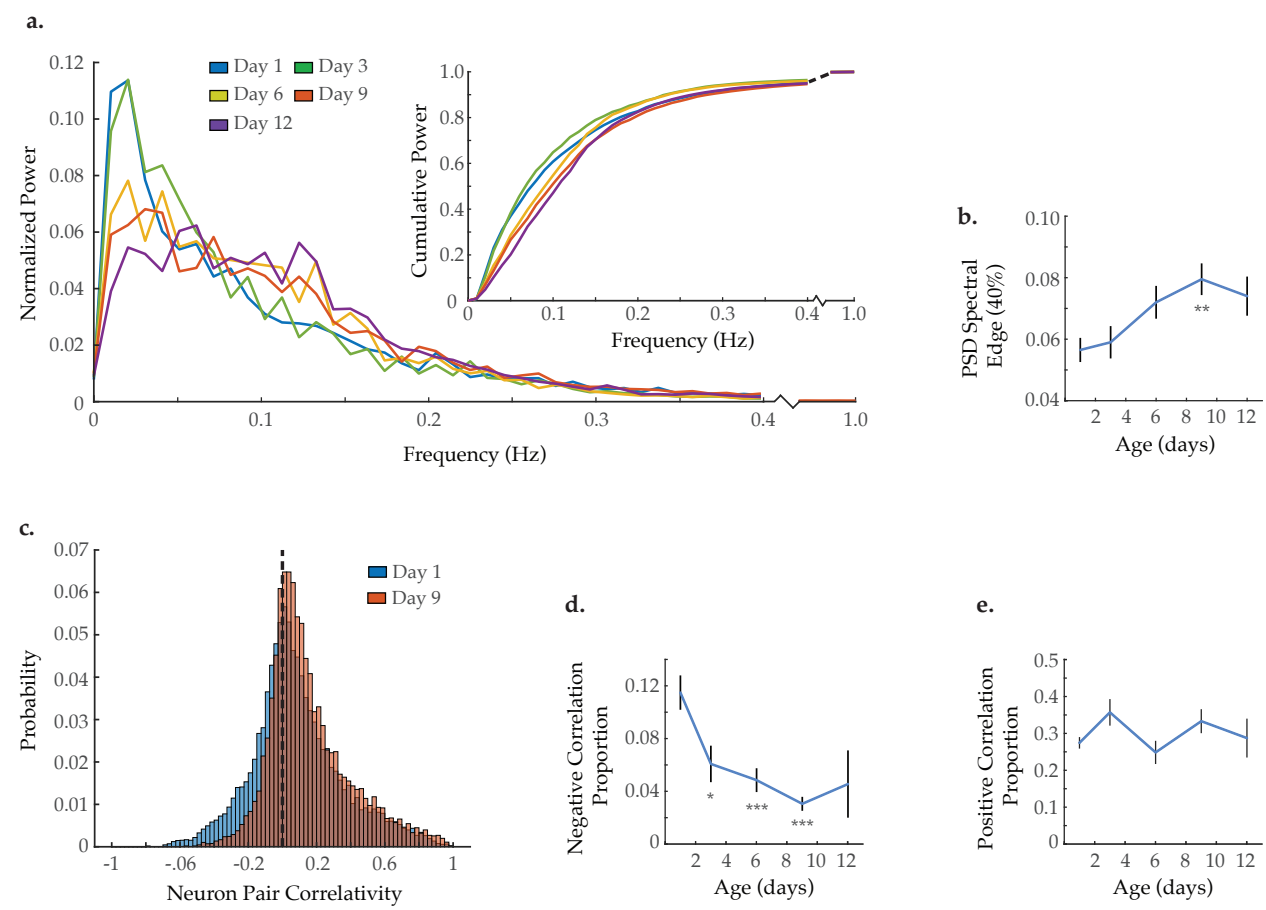
Figure 4

b.
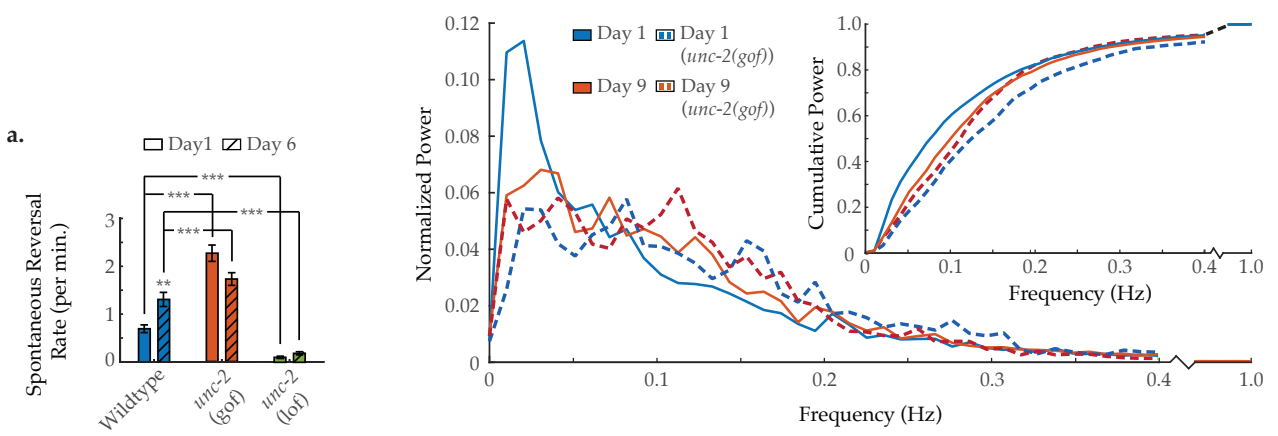

c. $\square$ Wildtype unc-2
(gof)
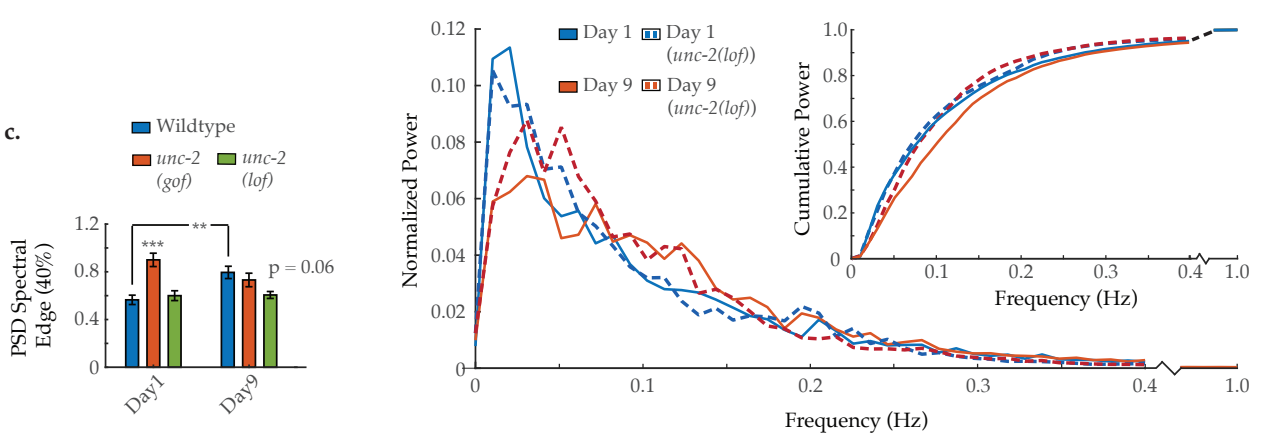

d.
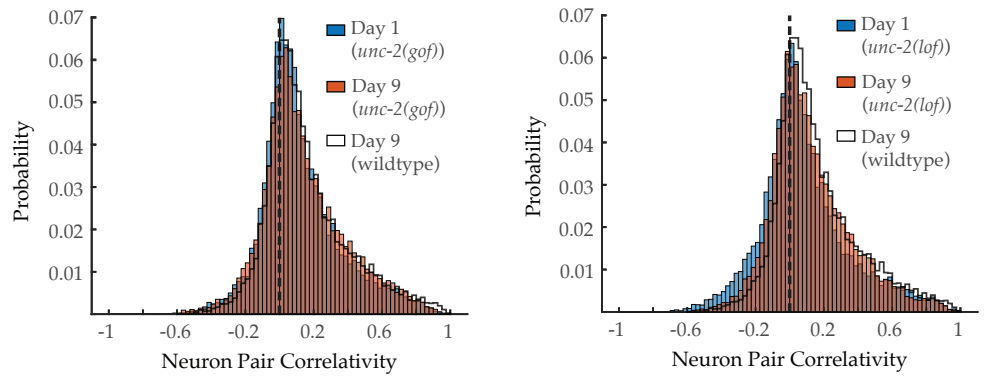

e.
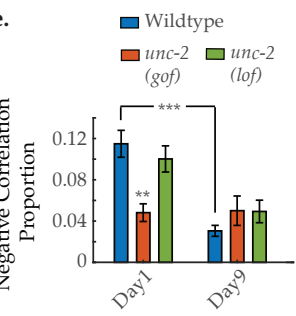

f.
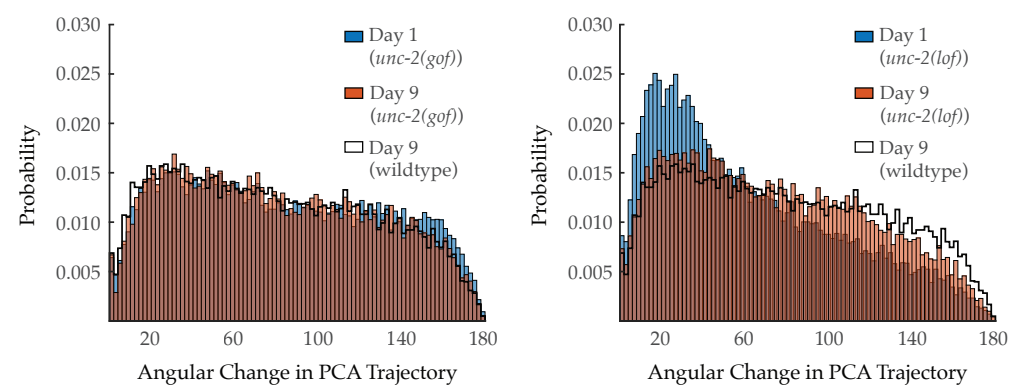
Figure 5

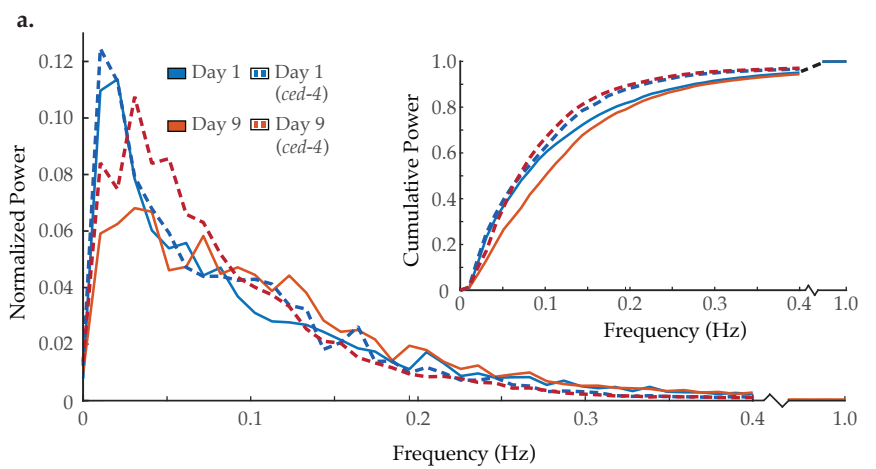

b.
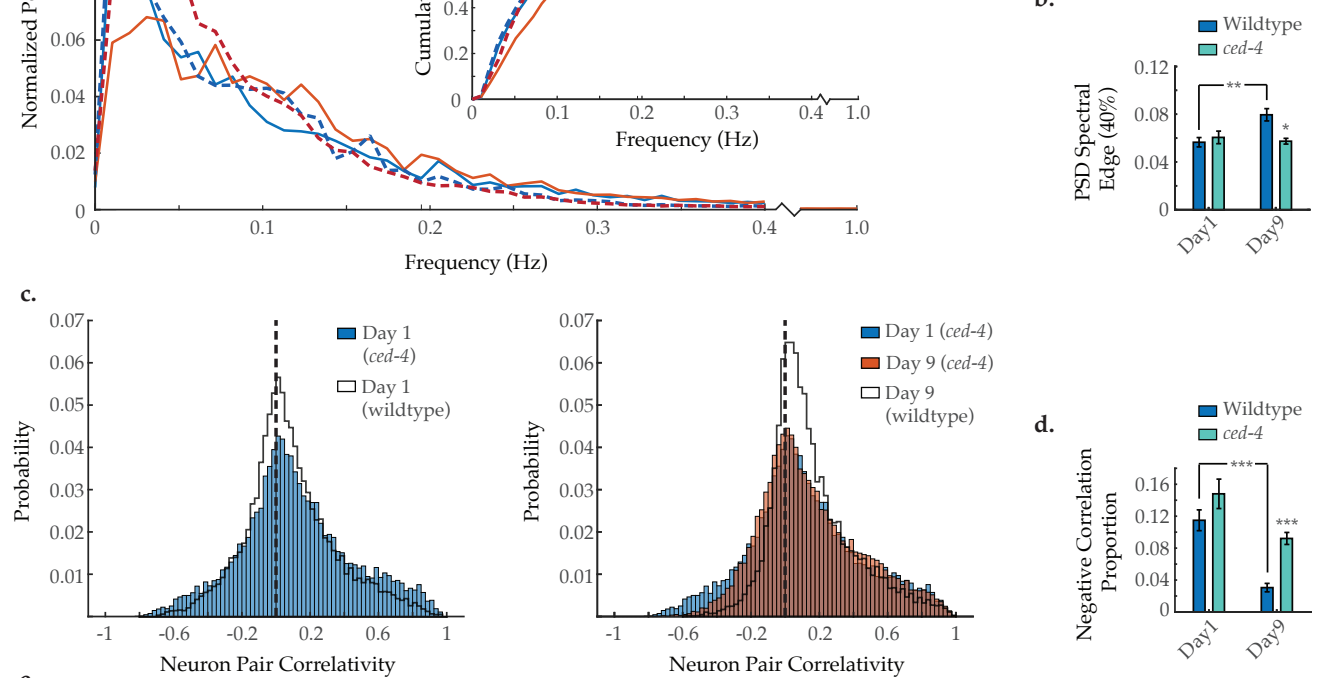

d.
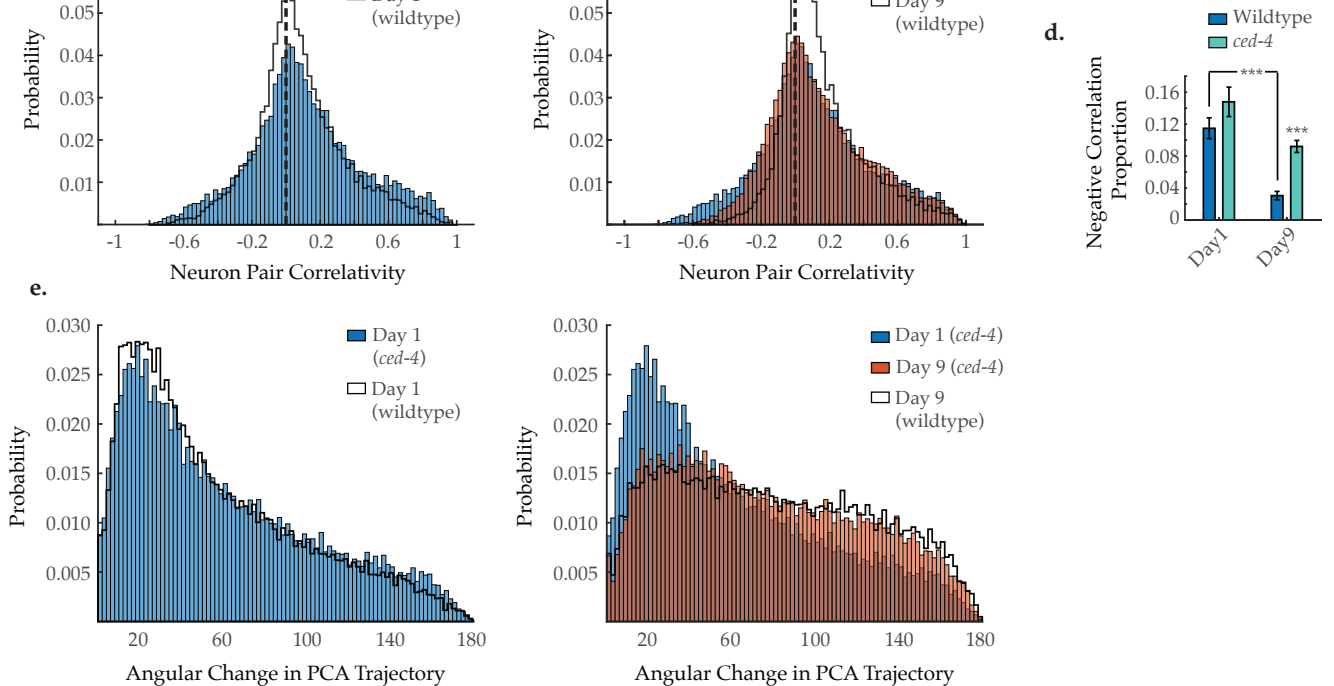
Figure 6

a.

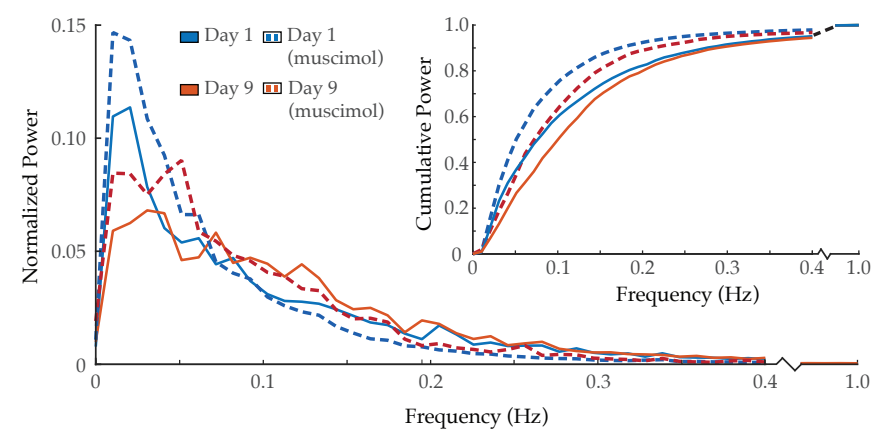

b.

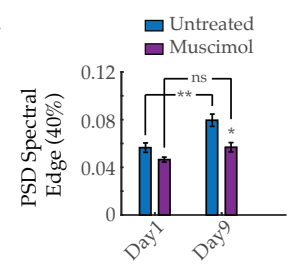

c.
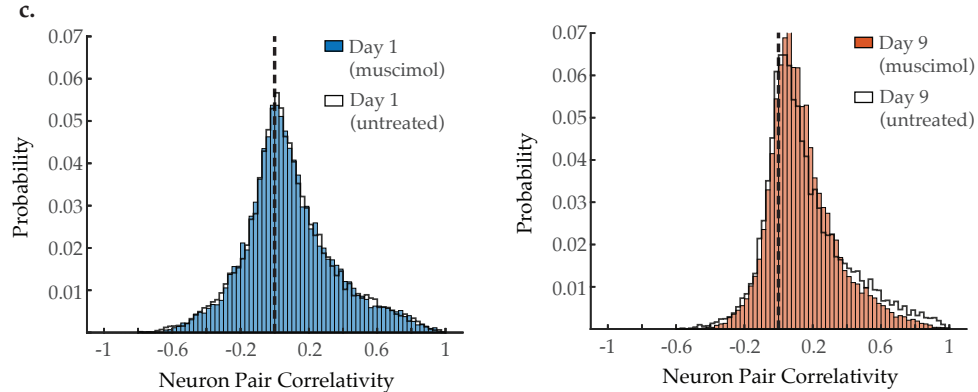

d. $\quad \square$ Untreated
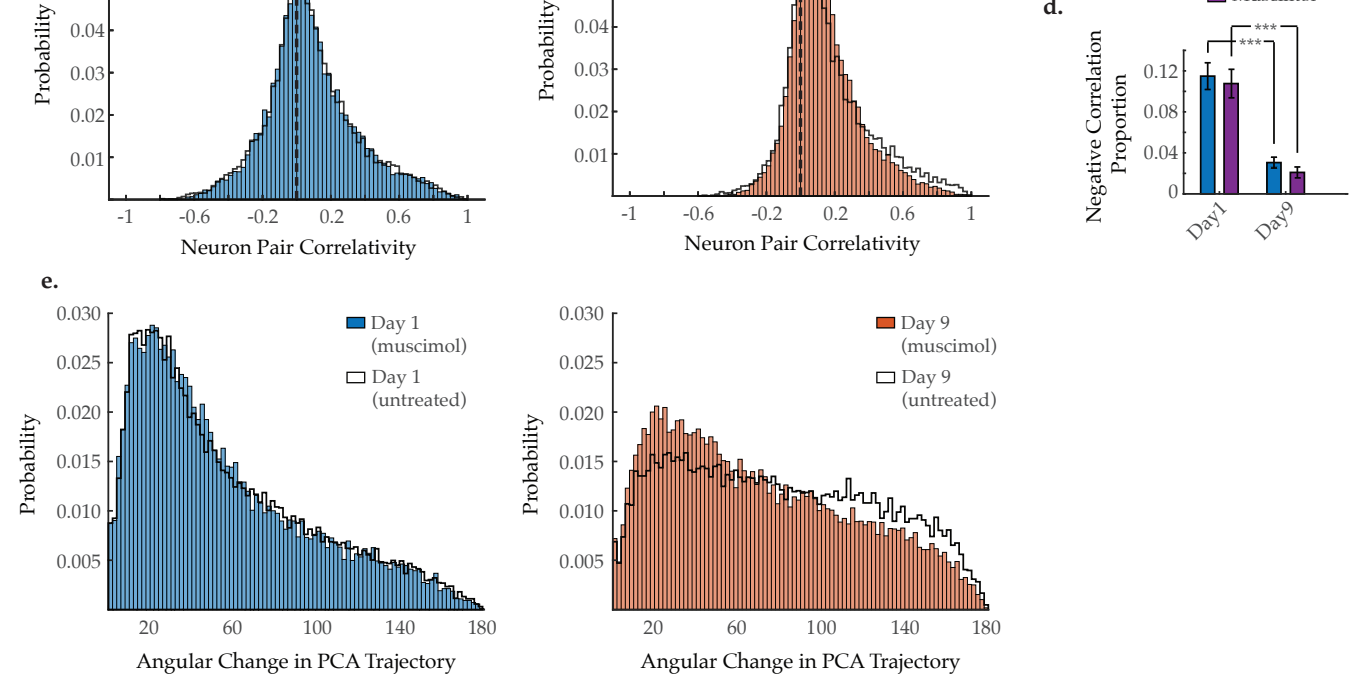
bioRxiv preprint doi: https://doi.org/10.1101/2021.07.07.451497; this version posted July 8, 2021. The copyright holder for this preprint (which was not certified by peer review) is the author/funder. All rights reserved. No reuse allowed without permission.

Figure 7
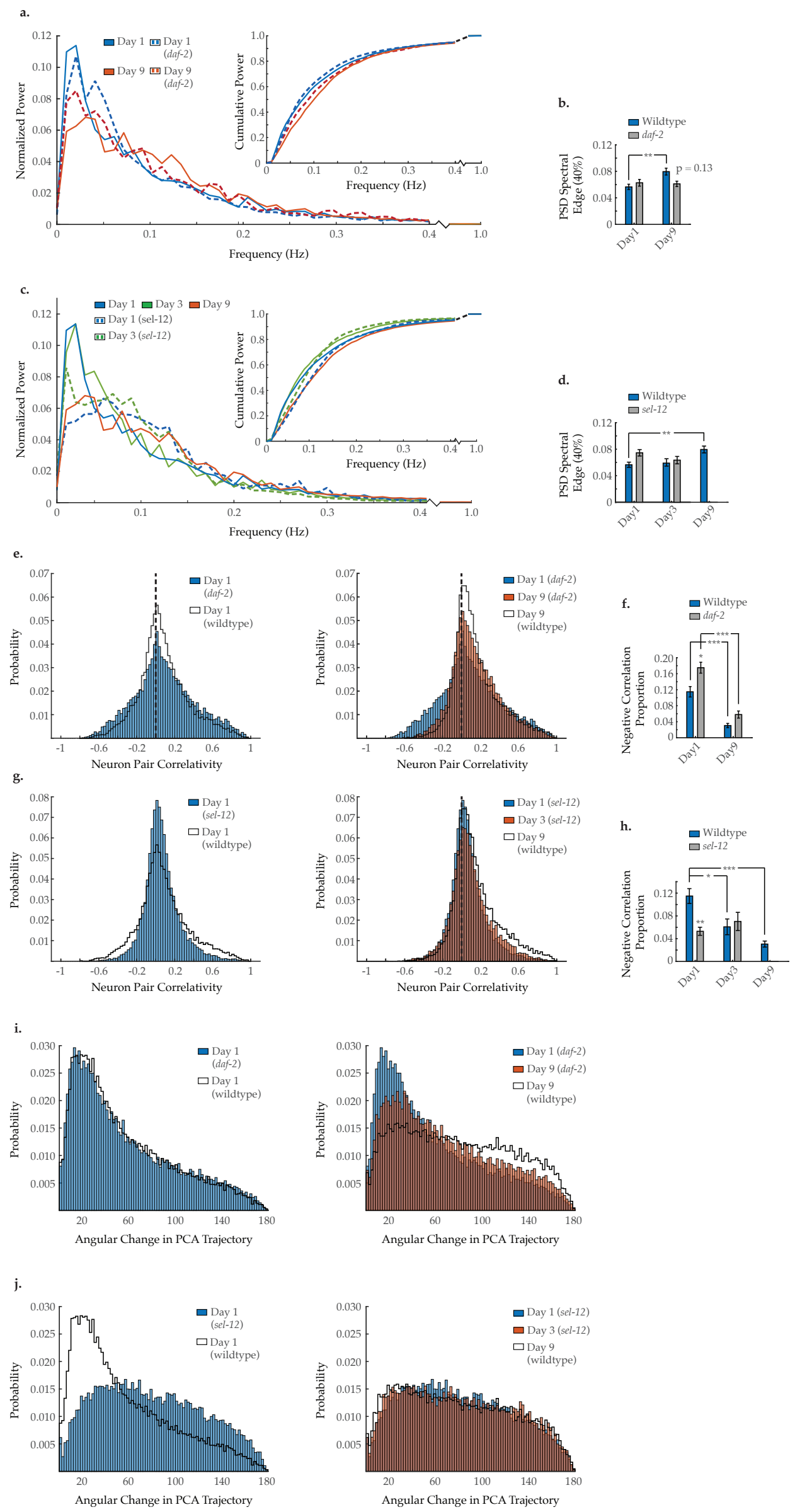
bioRxiv preprint doi: https://doi.org/10.1101/2021.07.07.451497; this version posted July 8, 2021. The copyright holder for this preprint (which was not certified by peer review) is the author/funder. All rights reserved. No reuse allowed without permission.

Figure S1

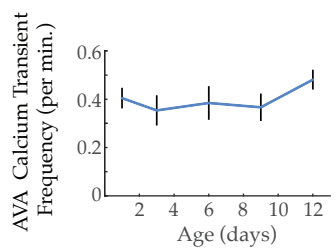

b.

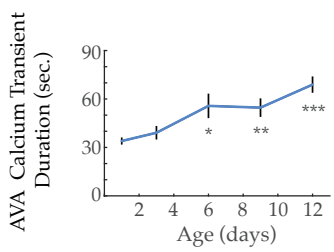


Figure S2

bioRxiv preprint doi: https://doi.org/10.1101/2021.07.07.451497; this version posted July 8, 2021. The copyright holder for this preprint (which
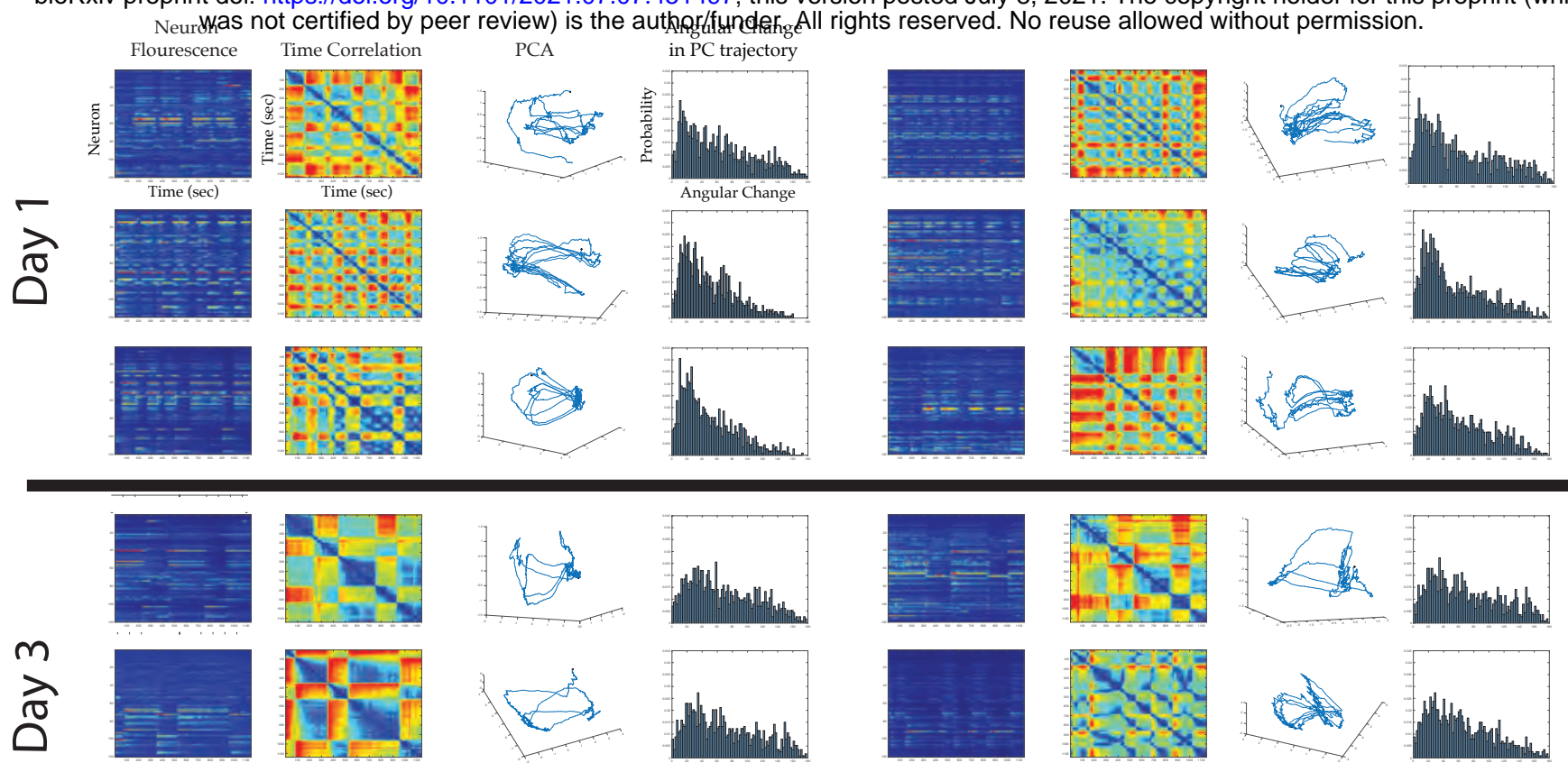

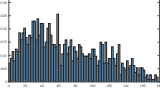
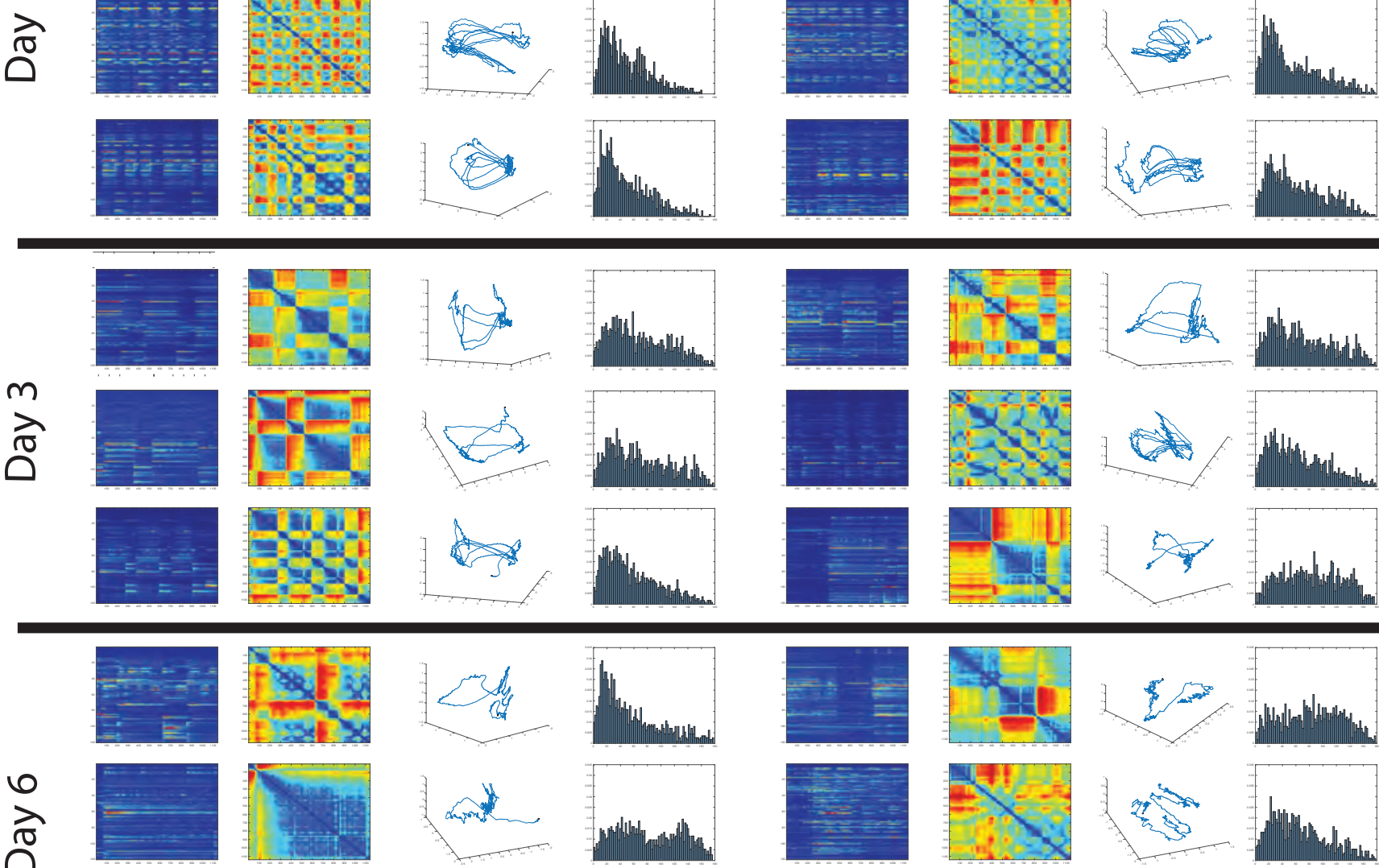

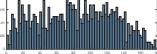
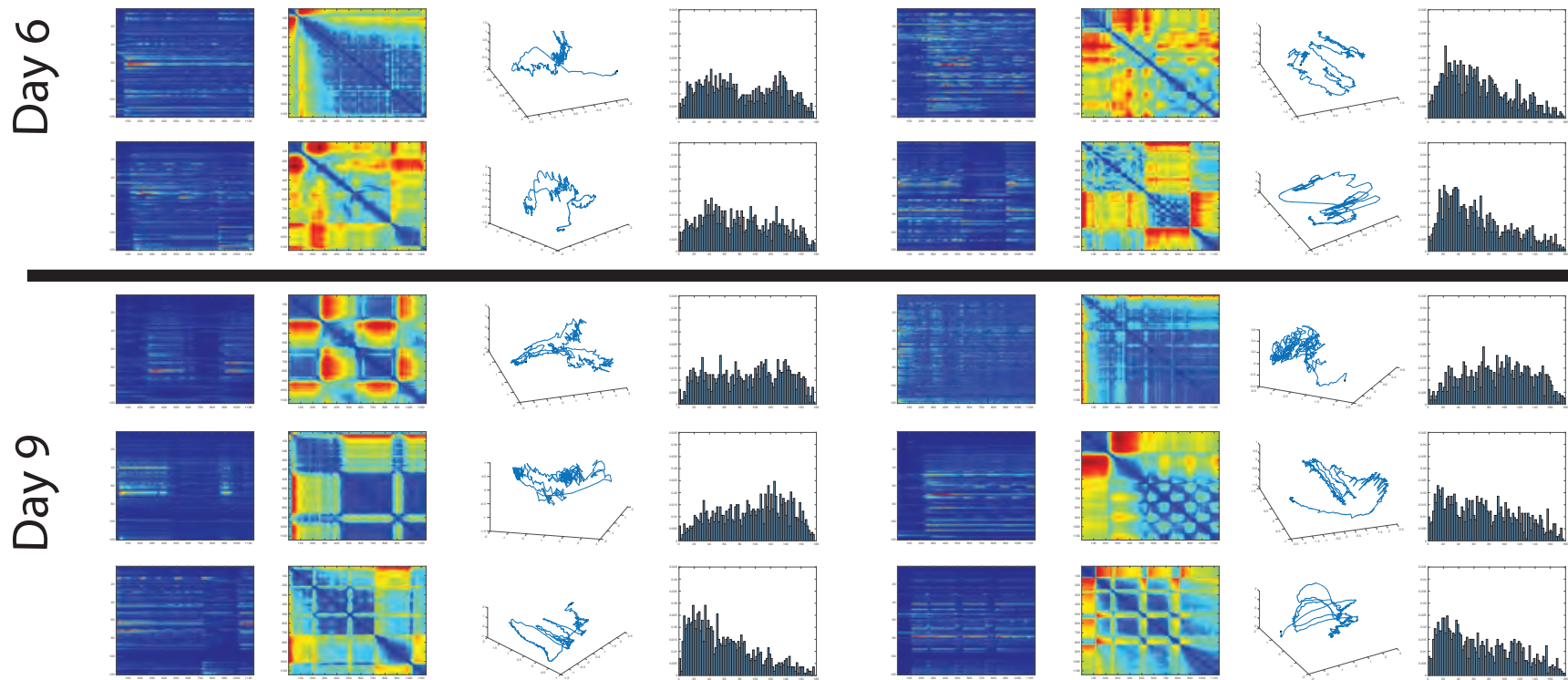

(n)
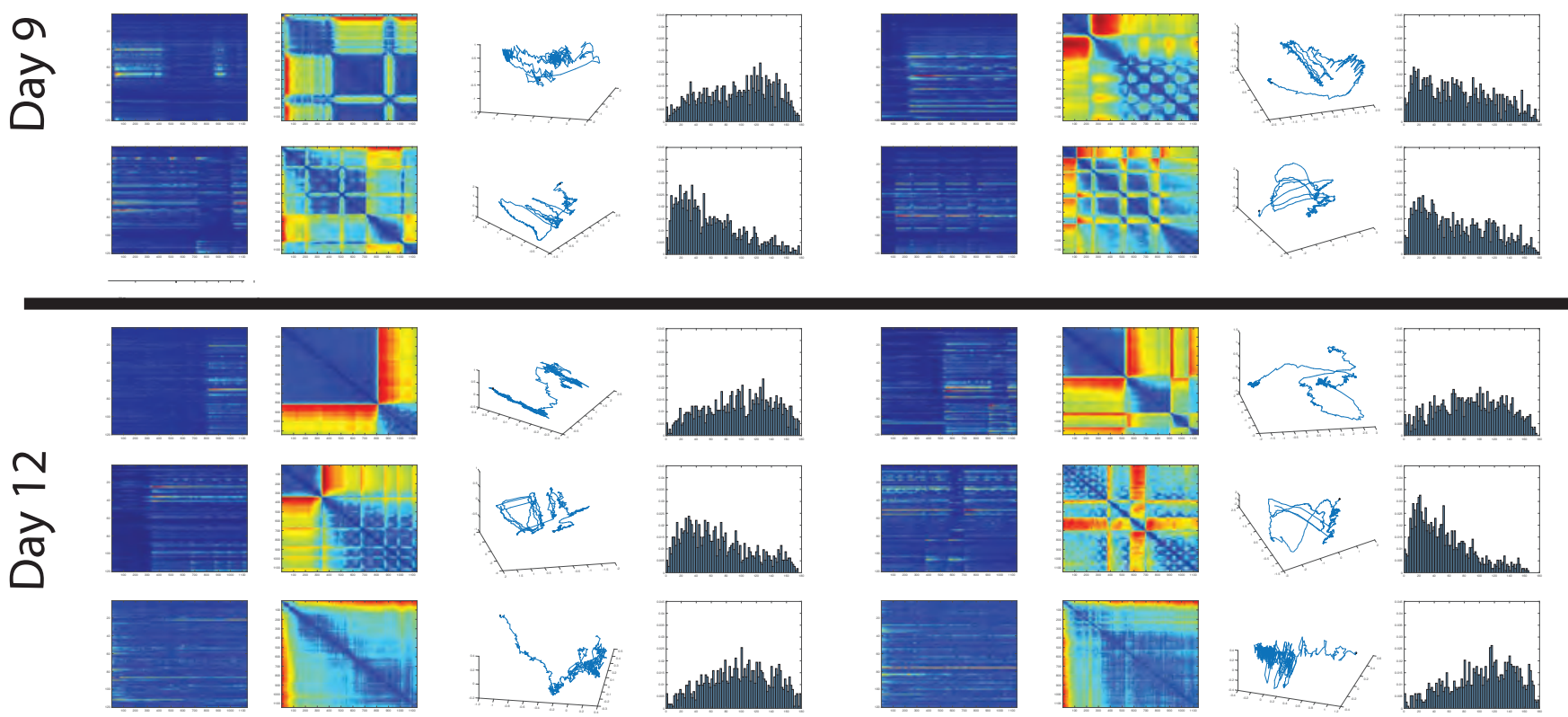

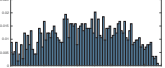
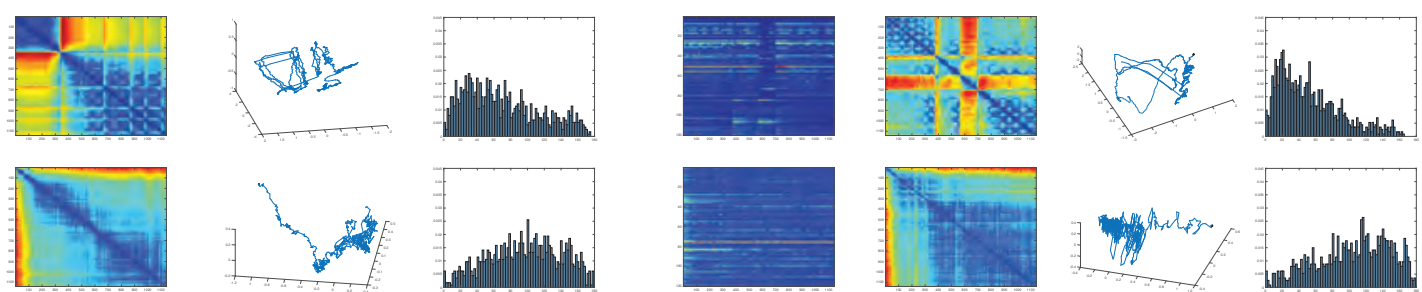
bioRxiv preprint doi: https://doi.org/10.1101/2021.07.07.451497; this version posted July 8, 2021. The copyright holder for this preprint (which was not certified by peer review) is the author/funder. All rights reserved. No reuse allowed without permission.

Figure S3
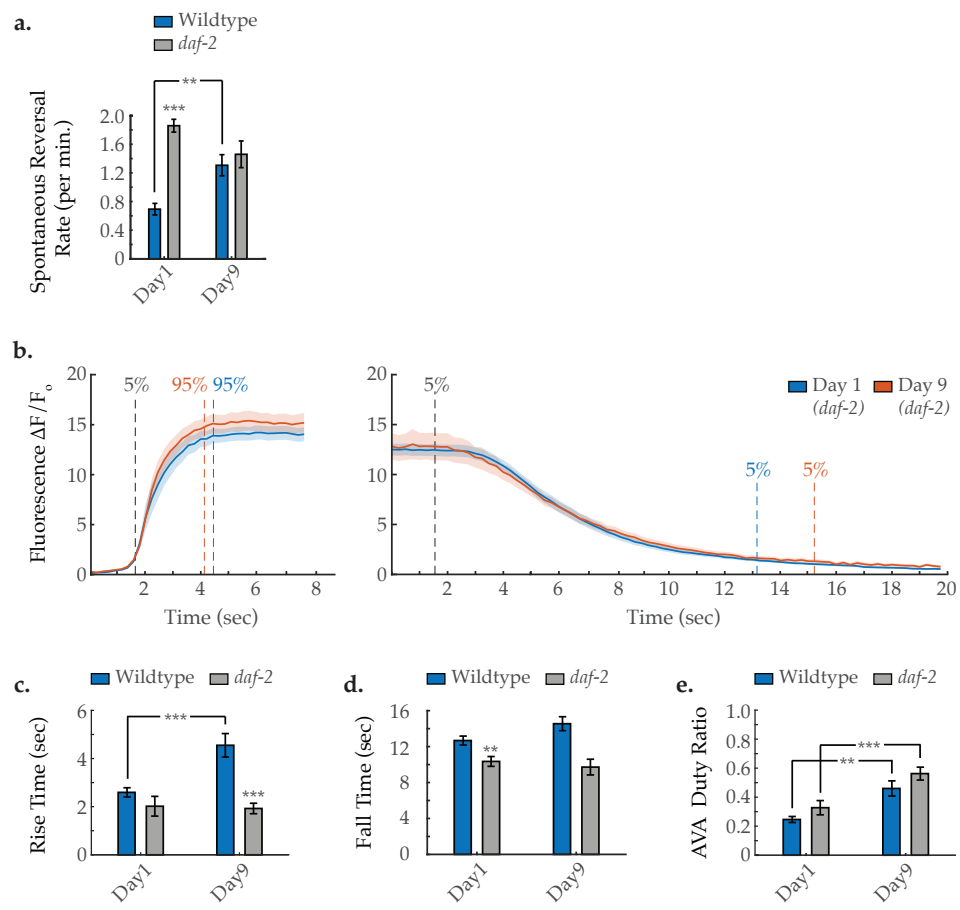